NBER WORKING PAPER SERIES

\title{
EXPANDING ACCESS TO CLEAN WATER FOR THE RURAL POOR: EXPERIMENTAL EVIDENCE FROM MALAWI
}

\author{
Pascaline Dupas \\ Basimenye Nhlema \\ Zachary Wagner \\ Aaron Wolf \\ Emily Wroe \\ Working Paper 27570 \\ http://www.nber.org/papers/w27570 \\ NATIONAL BUREAU OF ECONOMIC RESEARCH \\ 1050 Massachusetts Avenue \\ Cambridge, MA 02138 \\ July 2020
}

This research was made possible thanks to grants from Stichting Dioraphte and the Stanford Center for Innovation in Global Health. We gratefully acknowledge their support. These funders had no role in study design, data collection and analysis, decision to publish, or preparation of the manuscript. We thank Partners In Health Malawi for their partnership. The research protocol was approved by the Malawi National Health Sciences Research Committee and by the Stanford IRB. It is registered in the AEA RCT registry (AEARCTR-0002893). We thank Vivian Hoffmann and Michael Kremer for their inputs at the conceptual and design stage, and we are grateful to Ian Ross, Amit Dekel, Ismael Djima, Charlotte Pelras and Nina Rapoport for their insightful comments on our first draft. We also thank seminar participants at Stellenbosch University, DFID, and the Virtural Development Seminar for their helpful questions and suggestions. The views expressed herein are those of the authors and do not necessarily reflect the views of the National Bureau of Economic Research.

NBER working papers are circulated for discussion and comment purposes. They have not been peer-reviewed or been subject to the review by the NBER Board of Directors that accompanies official NBER publications.

(C) 2020 by Pascaline Dupas, Basimenye Nhlema, Zachary Wagner, Aaron Wolf, and Emily Wroe. All rights reserved. Short sections of text, not to exceed two paragraphs, may be quoted without explicit permission provided that full credit, including $\odot$ notice, is given to the source. 
Expanding Access to Clean Water for the Rural Poor: Experimental Evidence from Malawi Pascaline Dupas, Basimenye Nhlema, Zachary Wagner, Aaron Wolf, and Emily Wroe NBER Working Paper No. 27570

July 2020

JEL No. D10,D12,I11,I12

\begin{abstract}
$\underline{\text { ABSTRACT }}$
Using data from an 18-month randomized trial, we estimate large and sustained impacts on water purification and child health of a program providing monthly coupons for free water treatment solution (diluted chlorine) to households with young children. The program is more effective and much more cost-effective than asking Community Health Workers (CHWs) to distribute free chlorine to households during routine monthly visits. That is because only $40 \%$ of households make use of free chlorine, targeting through CHWs is worse than self-targeting through coupon redemption, and water treatment promotion by CHWs does not increase chlorine use among free chlorine beneficiaries. Non- use of free chlorine is driven by households who have a protected water source and those who report that chlorine makes water taste bad.
\end{abstract}

Pascaline Dupas

Department of Economics

Stanford University

579 Serra Mall

Stanford, CA 94305-6072

and CEPR

and also NBER

pdupas@stanford.edu

Basimenye Nhlema

Partners in Health

Neno

Malawi

bnhlema@pih.org

Zachary Wagner

RAND Corporation

1776 Main Street

Santa Monica, CA 90401

zwagner@rand.org
Aaron Wolf

Yale University

New Haven, CT 06520

United States

aaron.wolf@yale.edu

Emily Wroe

Partners in Health

Neno

Malawi

ewroe@pih.org

A randomized controlled trials registry entry is available at:

https://www.socialscienceregistry.org/trials/2893 


\section{Introduction}

Free access to essential health products such as vaccines, antimalarial bednets, or clean water is widely accepted as a cost-effective way to reduce the disease burden in lower income countries (Jamison et al., 2018). A large body of research supports this: take-up of preventatives is typically very low absent very large subsidies (see Dupas and Miguel, 2017, for a review). In some cases, full subsidies are not enough, however. Take-up rates of free flu shots in the United States are notoriously low (Milkman et al., 2011). Full immunization rates in Udaipur, India, plateaued at $18 \%$ even when reliable, free immunization camps were set-up in villages (Banerjee et al., 2010). Duflo et al. (2019) found no effect on sexually transmitted infection rates after Kenyan youths received a large supply of free condoms. Also in Kenya, Dupas et al. (2016) find that only $40 \%$ of households who get access to a free water treatment product use it.

Common strategies to boost take-up of free health services and products involve either information (e.g. mass media information campaigns), financial incentives, or nudges (e.g. SMS reminders). Such strategies are common in higher-income countries. In lower-income countries, where target populations are often rural, dispersed, and may not all have access to a radio or a phone, such strategies can be prohibitively expensive. Even when they are feasible, their impacts on take-up appear limited. A meta-analysis of 10 recent studies of text message reminders in the United States, Kenya, Nigeria, Guatemala, and Zimbabwe find that they increase childhood vaccination by $13 \%$ on average (Mekonnen et al., 2019). Full immunizations rates increased from $18 \%$ to $36 \%$ when in-kind-incentives were provided to parents in the Udaipur experiment of Banerjee et al. (2010)-an impressive doubling, but even with incentives more than half of children did not receive full immunization. What else can be done, then, to increase take-up of free preventative health services among the rural poor?

An increasingly prevalent model for health outreach in rural areas of lower-income countries is

the Community Health Worker (CHW) model. In this model, lay members of the community work (either for pay or as volunteers, depending on the model) in association with the local health care system, typically visiting households at home, providing basic diagnostic services, and referring sick patients to facilities as well. Recent studies have found that properly compensated CHWs can effectively be used to expand access to curative products (Wagner et al., 2019) 
and reduce child mortality (Björkman-Nyqvist et al., 2019). Whether CHWs are effective at encouraging households to adopt specific health practices remains an open question, however. As members of the community, they might have useful information about which households are likely to reap higher returns from investing in prevention. They also have the ability to make recurrent visits, monitoring and nudging households to be consistent in their preventative behavior.

We study the potential complementarity between CHWs and full subsidies in the context of safe water access in Malawi. In a region with a well-established NGO-supported CHW program, in which CHWs work under the government's Health Surveillance Assistants (HSAs) and are assigned 20 to 30 households each to monitor, we randomly assign households to receive either 18 months of coupons for free chlorine solution redeemable at local shops, free monthly chlorine deliveries by CHWs, or to be in a control group. We cross randomize CHWs to incorporate active Water, Sanitation, and Hygiene (WASH) education into their monthly household visits to test if this increases chlorine take-up and/or helps sustain chlorine use over 18 months. In a neighboring region with only the HSA program (no NGO-supported CHWs), we randomize households between the coupon program and a control group.

We find that the coupon program increases verified chlorine usage rates during spotcheck visits from $5 \%$ to $30 \%$. This effect is constant across the 18-month period of follow-up. The share of households with verified chlorine usage during at least one of the two followup visits increases from $6 \%$ to $46 \%$. This level of usage seems to be the ceiling, however: none of the CHW-based interventions have additional impact. To start, the impact of the coupon program is larger in the region without a $\mathrm{CHW}$ program. In the region with a $\mathrm{CHW}$ program, the impact of the coupon program is identical whether or not the CHW was randomized into being trained to promote WASH. Finally, and most surprisingly, relying on CHWs to deliver free chlorine to all households during monthly visits does no better than the coupon program at improving chlorine usage. If anything, it does slightly worse due to imperfect compliance among CHWs, combined with imperfect targeting.

The large increase in chlorine usage under the coupon program translates into substantial child health impacts. Caretaker-reported incidence of diarrhea, fever, and vomiting - all symptoms of water-borne infections - go down by 20 to $25 \%$ in the coupon group within 6 months and 
remains lower over the entire 18 month follow-up period. The health effects are smaller under the home delivery by CHW program. This is due to poorer targeting by CHWs than through the coupon program. Households who do not receive the subsidy from CHWs are disproportionately vulnerable to water-borne diseases. In contrast, those vulnerable households are significantly more likely to avail themselves of free chlorine through coupon redemption.

These results suggest that (non-incentivized but well compensated) CHWs are neither complement nor substitute with subsidies: they do not increase take-up absent subsidies nor do they increase take-up of subsidies. Using CHWs as behavior change communication and/or nudge agents appears to be an inefficient use of their time in contexts where households are already informed of the importance of clean water during prenatal and postnatal care. ${ }^{1}$

Overall, less than half of households in our sample ever use chlorine they receive for free, even in the presence of a WASH promotion program. That means that blanket free dispensing leads to substantial wastage. We test whether CHWs can be used to effectively target the subsidies to households who have an interest in the product. After CHWs in the home delivery group had been doing deliveries for 8 months (during which they received enough chlorine to distribute to their entire community), we randomized a "rationing" treatment: while some CHWs continued as before (no rationing), others received only $60 \%$ as many chlorine bottles as households in their community (medium rationing) and some only 40\% (strong rationing). Rationed CHWs were explicitly asked to try to deliver the chlorine bottles to households most likely to use the chlorine to treat their water. ${ }^{2}$ We find that fewer households received deliveries after the rationing starts, but the share of households with verified chlorine usage did not decrease. This suggests that CHWs have or can easily acquire information about the households they visit regularly, and can use it to target subsidies to users. Targeting on underlying need is less clear, however: the health effects of the home delivery by CHWs become indistinguishable from zero in the rationing treatment.

Overall, using CHWs to target chlorine subsidies does no better than the coupon program. That is because only a small number of households go through the hassle of redeeming coupons

\footnotetext{
${ }^{1}$ Over $95 \%$ of women in the Malawi Demographic and Health Surveys reported using formal postnatal care for their most recent birth.

${ }^{2}$ There were no incentives for CHWs under the rationing treatment.
} 
for free chlorine without using the product afterward, and those with higher returns to product use are more willing to pay the hassle cost, so self-targeting under the coupon scheme is very good. There are, however, some domains where self-targeting may not work or where a coupon scheme may not be an option. In such cases, using CHWs as information gathering agents could be very useful - e.g. to identify households that need to go for a TB screening test, do not comply with their ARV drug regimen, etc. We note that incentives for CHWs to gather this information may be necessary, however. As noted above, households in most need of chlorine were somewhat less likely to receive routine $\mathrm{CHW}$ visits to start, in part because they are further away.

We view our study as making two main contributions. First, we demonstrate large and sustained health impacts of a program that provides coupons for free chlorine to households with young children. While Dupas et al. (2016) have previously demonstrated, in the Kenya context, the effectiveness of coupons as self-targeting micro-ordeals, that study only looked at short-term usage and did not include health outcomes. We find that the targeting results of Dupas et al. (2016) in Kenya are sustained over time, hold in Malawi 10 years later, and that effects on health can be substantial, for a very low cost. This suggests that governments facing a nontrivial water-borne disease burden may want to consider embedding a coupon program into their existing well-baby schemes, the same way most governments around sub-Saharan Africa have by now put in place free bed net distribution programs through prenatal and child clinics (Dizon-Ross et al., 2017). New parents could easily receive a booklet of coupons at the time they come for prenatal care, deliver a child and/or bring a child for immunization. Coupons could then be redeemed at local shops or pharmacies or health facilities, depending on the procurement system that can be set-up. Physical coupons may even become unnecessary as countries adopt biometric IDs-a reform currently underway in Malawi, Cote d'Ivoire, Ghana, and many others.

Second, we contribute to the growing literature on CHW programs. CHW programs across the globe are highly heterogeneous. Those evaluated to date have been a bundle of multiple interventions, as CHWs are typically asked to do many things: provide information and encouragement; provide diagnostic help and refer serious cases to the health facility; deliver essential health products, sometimes for free and sometimes at a fee. It is unclear whether the impact of 
successful programs stems from the information provision, the encouragement, the surveillance, or the delivery component. Our results suggest that information provision and encouragement may only play a limited role. There is however scope for the surveillance component to be very large when the effectiveness of the social planner's intervention depends on private information about households, private information that the CHWs appear to be effective at discerning, though they may not always have the incentives to do so.

The remainder of this paper proceeds as follows. Section 2 provides some background on waterborne disease prevention and the study context. Section 3 lays the theoretical foundation for the experimental design. Section 4 presents the study design and data. Section 5 presents the results and Section 6 discusses cost-effectiveness. Section 7 concludes.

\section{Study Background}

\subsection{Unclean Water, Disease Burden, and Chlorine Access}

Globally, an estimated 1.9 billion people lack access to clean water, meaning that they use either an unimproved water source or an improved source that is contaminated with fecal matter. ${ }^{3}$ Unclean water leads to water-borne diseases, chief among them diarrhea. Diarrheal disease is the second-leading cause of childhood mortality. In countries that cannot afford to provide piped water to dispersed, rural households, point-of-use water-treatment by households can reduce reported child diarrhea by 29\%. ${ }^{4}$ Chlorine disinfects drinking water against most bacteria and protects it from re-contamination. Drinking water treated with dilute chlorine is safe, even if widely used over a long period. ${ }^{5}$ Additionally, chlorinated water does not need to be boiled or filtered, which saves users time and money spent on fuel, while conserving environmental resources. The problem is that current usage rate of chlorine among rural households in SubSaharan Africa is low - at baseline in 2017, in our study context of Southern Malawi, it was only

\footnotetext{
${ }^{3}$ https://www.who.int/water_sanitation_health/publications/hwt-scheme-round-1-report.pdf

${ }^{4}$ See Wolf et al. (2018), Arnold and Colford (2007), Clasen et al. (2007), Fewtrell et al. (2005) for reviews of this literature.

${ }^{5}$ Chlorination has a long history of use, and became a standard of water treatment during the first half of the 20th century in Europe and the United States. Long-term experience with the substance in piped water supplies has made clear that its use is safe. The European Commission, U.S. Environmental Protection Agency, and World Health Organization support the use of chlorine for water treatment as long as it is used within acceptable dose ranges.
} 
5\%. Finding ways to increase chlorine use among rural households is an outstanding challenge for the global health community as it strives to achieve the Sustainable Development Goal of universal safe water by 2030 .

In areas where a single water source is used by many households, communal infrastructure (dispensers placed at water sites) have been proposed as a delivery mechanism with the potential to increase chlorine usage (Kremer et al., 2011). But in areas where the number of users per water source is low, the current standard approach is to encourage populations, via promotional campaigns, to purchase and use chlorine bottles from local shops, and take-up remains low under this model. For example, in Malawi and Kenya, where the NGO PSI spent over a decade promoting a diluted chlorine product locally called "WaterGuard liquid" as well as another point-of-use water treatment product locally called "WaterGuard powder", the usage rate of any WaterGuard product at the onset of this study was estimated by PSI at $12 \%$ in Malawi (2017), and $16 \%$ in Kenya. ${ }^{6}$

An alternative approach would be free distribution to parents of young children through health centers. This approach was tested in an experimental study in Kenya in 2007, and was shown to drastically increase usage-but also to generate wastage if not all recipients of subsidized chlorine use it for water treatment: Dupas et al. (2016) found that only around 40\% of households who had received enough WaterGuard for a year were treating their water with it during a 6-month spotcheck visit. One mechanism proposed to reduce wastage is to require that households pay some hassle cost in order to get the free water treatment product. Dupas et al. (2016) show that coupons that required retrieval of free chlorine from a local shop achieved the same level of chlorine use as free home delivery at a considerably lower cost, since households not interested in using the product do not bother redeeming coupons but feel compelled to accept the home delivery.

While targeting via coupons improves the cost-effectiveness of free distribution, it falls short of achieving widespread coverage of safe water. How can the effectiveness of free distribution schemes (be it home delivery or coupon programs) be enhanced? As numerous countries scale

\footnotetext{
${ }^{6}$ The Malawi figure comes from this report: http://www.psi.org/country/malawi; we compute the figure for Kenya as follows: 27 million month-long treatment units sold (based on https://www.pskenya.org/ wp-content/uploads/2019/04/PS-Kenya-2018-Annual-Report.pdf) for a country with 14 million households: 27 million/12 months/14 million households=0.16.
} 
up the use of Community Health Workers (CHWs), one potential approach is to rely on CHWs to promote the use of water treatment. There are three main mechanisms through which CHWs could increase the returns to dollars invested in water treatment subsidies. First, CHWs could increase take-up through behavior change communication (BCC); namely, explaining to households the returns to safe water and how to treat water with chlorine. Second, CHWs could increase take-up through a reminder effect: CHWs are typically tasked with visiting households monthly, and these monthly visits could act as a reminder for households to treat their water. Third, CHWs could use their local information to target the subsidies to households most likely to put the chlorine product to good use.

\subsection{WaterGuard/MadziGuard}

In this study, we focus on efficient distribution of a product called WaterGuard, a dilute chlorine solution (1.5\% sodium hypochlorite) that was produced and socially marketed by Population Health Services (PSI) in Malawi until December 2018. Production of this solution was transferred to Pharmanova Malawi Limited from December 2018 onwards (about 12 months after our study began) under the brand name MadziGuard. ("Madzi" means water in the local language. For simplicity, for the rest of the paper and in tables and figures we use only the term "WaterGuard" to refer to the treatment solution). One capful of the product contains enough chlorine solution to treat 20L of visually clean water (two capfuls is recommended for water that is cloudy or dirty-looking). The WaterGuard product was recognized throughout the study area: $51 \%$ of respondents reported having ever used WaterGuard at baseline (see Table 1). Despite this, water tests show that only $4.6 \%$ of all respondents had treated their water with chlorine at baseline.

\subsection{Government's Health Surveillance Assistants}

The Malawi Ministry of Health (MoH) has a cadre of Health Surveillance Assistants (HSAs) linked to primary-level health facilities. HSAs are tasked to deliver a range of services to their assigned communities, including information on hygiene and sanitation; immunizations; ante- 
natal and postnatal care education; and nutrition counseling, among other things. ${ }^{7}$ During cholera outbreaks, HSAs can be asked to provide free water treatment solution to households. Households in our sample report some non-trivial amount of interactions with HSAs on water. $51 \%$ report that they ever received water management advice from a HSA (Table 1). ${ }^{8}$ Distribution of water treatment solution by HSAs is relatively rare however, with only $4 \%$ of households reporting that HSAs regularly dispenses WaterGuard and less than $8 \%$ having ever received free chlorine from an HSA.

\subsection{Partners In Health CHW program}

We worked with the CHW program run by Partners In Health (PIH). PIH works with the MoH to provide comprehensive care for about 150,000 people in the rural district of Neno in Southern Malawi. PIH complements its clinical services with outreach programs including CHWs. The CHWs employed by PIH act as 'foot soldiers' to the MOH's cadre of HSAs and are able to support the HSAs in the community and in the home. Whereas HSAs in Neno have a ratio of about 1:2000 population, the CHWs have a ratio of about 1:150.

CHWs are trained and paid 17,000 Malawian Kwacha (MWK) a month (about 23 USD-for reference, the annual GDP per capita in Malawi in current USD is 340). They are supposed to visit each household in their catchment area once per month, checking on the health needs of the entire household, and referring family members to clinics for care. Topics covered during home visits include child nutrition, maternal health, and management of HIV, Tuberculosis and non-communicable diseases. Summary statistics on CHWs in the study are shown in Appendix Table A1. CHWs are 37 years old on average and $65 \%$ female. About $65 \%$ completed at least primary school (compared to only $45 \%$ of the population in their catchment area). CHWs are responsible for about 27 households and work about 10 hours per week on CHW activities. Over 75\% have a job in addition to their CHW role and CHW work is the primary source of income for $43 \%$ of CHWs. A Senior CHWs cadre exists who have added mentoring responsibilities vis-a-vis other CHWs.

\footnotetext{
${ }^{7}$ Government of the Republic of Malawi. 2009. Health Surveillance Agent Training Manual Facilitator's Guide (PDF, 1.7 MB). Lilongwe, Malawi: Ministry of Health.

${ }^{8}$ The likelihood of having received such advice is lower for those who live farther away from a trading center: each kilometer reduces it by 2 percentage points.
} 


\section{Conceptual Framework}

In many contexts, a principal would like an agent to undertake an action which cannot be routinely observed. One tool at the disposal of the principal is to provide the agent an input which is complementary to the desired action. In our setting, the principal subsidizes chlorine solution to promote water treatment among households with young children. We consider a principal who values the health benefit of a health product, non-health utility, and alternative uses of funds. Denote $b_{i}$ the health benefit when $i$ uses the health product appropriately, $z$ the dollar value of a unit of health to the principal, and $h_{i}$ the binary variable indicating whether $i$ uses product appropriately. The household's non-health utility is $u_{i}$. Letting $S$ denote the total cost of subsidies to promote use of the health good and $\lambda$ the marginal cost of public funds, the principal's payoff is:

$$
\sum_{i}\left(z * b_{i} * h_{i}+u_{i}\right)-\lambda S
$$

Because some households may accept a subsidized health good but not use it for a health purpose (or not use it at all), we distinguish between "takers" and "users", where the former includes all of those who take the good, regardless of whether and how they use it, and the latter refers to those who use the health good for the health purpose. From the perspective of the principal considering whether to introduce a subsidy or other measures to increase usage of the health product from some baseline level, a subsidy is preferred if the value of health benefits generated by marginal users of the product, plus any changes in the non-health utility of marginal and inframarginal users, exceed the opportunity cost of the subsidy spending:

$$
\left(z b_{\text {mar }}+d u_{\text {mar }}\right) u s e_{\text {mar }}+d u_{\text {inf }} u s e_{\text {inf }}>\lambda s\left(\text { take }_{\text {inf }}+\text { take }_{\text {mar }}\right)
$$

On the left-hand side of this inequality is the per-household benefit (suppressing subscript $i$ ) to the principal of introducing the subsidy. The health benefit of the policy is the individual value of this benefit, $z$, multiplied by $u s e_{m a r}$, the proportion of households induced to use the product under the policy. The non-health utility benefit to such households is represented by $d u_{\text {mar }}$. Households that already use the health product for the health purpose experience no additional health benefit, but may experience an increase in non-health utility $d u_{\text {inf }}$ through the reduced monetary or effort cost of obtaining the product. The proportion of such households is represented by $u s e_{i n f}$. On the right-hand side is the principal's cost of the potential policy: 
$s$ is the cost of the policy per household that takes up the health product. The terms take $e_{i n f}$ and take mar represent the proportion of households already taking the health product before the change and those newly induced to take it, regardless of whether they use it for the health purpose.

This simple framework helps highlight the potential tradeoffs across possible subsidy policy designs. If $u s e_{\text {mar }}<t_{a k e}$ mar , i.e. the subsidy policy induces some people to take the input but they end up not using it appropriately, then the policy leads to errors of inclusion, which are costly. Reducing the gap between $u_{s e}$ mar and take mar will increase cost-effectiveness. One potential way to reduce this gap is to encourage takers to put the product to its intended health use, through information or behavioral change communication. Alternatively, the gap between $u_{s e} e_{m a r}$ and take $_{\text {mar }}$ can be reduced through a subsidy delivery mechanisms that screens out non-users. Our study studies both margins: we study a program that attempts to increase usage among takers (WASH encouragement by CHWs) as well as two screening mechanisms: self-screening through coupon redemption, and screening by CHWs.

The effectiveness of the policy will also depend on $b_{\text {mar }}$, the health return to product use for households induced by the subsidy to take and use the input. As shown in Table 1, households in our sample are heterogeneous in the baseline safety of their water, with about half of households already benefiting from a protected source, suggesting that a large share of the sample may have fairly low $b_{\text {mar }}$. A subsidy delivery mechanism that helps screen out those households will be more cost-effective. Whether it maximizes welfare depends on the value placed on health and on the cost of the product. This highlights the importance of comparing the targeting properties of possible subsidy policies in terms of the underlying need for water treatment, not only usage.

Finally, the framework highlights that the higher is the baseline take-up of the product $\left(\operatorname{take}_{\text {inf }}\right)$, the higher is the program cost. In our context, as shown in Table 1, baseline usage is below $5 \%$, so the potential for subsidies to be cost-effective is high. 


\section{Study Design and Data Collection}

\subsection{Study Setting}

The study took place in the region that was formerly known as Mwanza District in Southern Malawi. In 2003, the district was split into two districts, Neno and Mwanza, under the decentralization program. The two neighboring districts are relatively poor and rural. PIH works only in Neno; there is no CHW program in Mwanza. Water-borne diseases are a leading cause of death for children under-5. A representative survey in 2015 shows that over $20 \%$ of children in the Southern region of Malawi had a case of diarrhea within the prior two weeks (NSO and ICF, 2017). This may stem from the fact that very few households have piped water (less than $3 \%$ in the 2015 survey and $4 \%$ in our baseline sample).

\subsection{Sample}

We conducted a household listing in November 2017 in the selected study areas, enumerating every household, for a total of 10,576 households in Neno and 3,946 households in Mwanza. Among those, households with a child under the age of 6 were considered eligible for the study, and a subset of those eligible were sampled for the study.

CHWs in Neno are assigned to specific clusters of households. To sample households for the study, we stratified by these clusters. Specifically, we randomly sampled 6 eligible households from clusters with more than 15 households, and 4 eligible households from clusters with less than 15 households. To mimic this sampling strategy in Mwanza district, where there is no CHW program, we created 110 areas of geographically proximate households and randomly sampled 4 households from each. In addition to the main sample, 4 households from each area in both Neno and Mwanza were added as "backup" households: If, during baseline data collection, a household was no longer available, ineligible, or turned out to be outside the catchment's area of the CHW cluster we thought they were in during the listing, the household was replaced with one of these backup households. This backup households would inherit the treatment status of the original.

A baseline survey was conducted between March and April of 2018. A total of 2,012 households 
from the main list were successfully surveyed and incorporated into the study. 301 further households from the backup lists were used as replacements, giving us a final sample of 2,313. Table 1 shows the demographics and water related characteristics of our sample. Since we sampled households with young children, the sample is young, with mothers aged 30 on average. The average household has just over 5 members. $65 \%$ of households use a borehole as their primary source of drinking water and $30 \%$ use an unprotected source such as a spring. While the sample appears quite knowledgeable about Waterguard and its effectiveness (71\% think that WaterGuard makes water safe), and although about half report ever using it, there is widespread concern that it makes the water taste bad ( $46 \%$ of respondents). At the time of the baseline survey, only $4.6 \%$ of households had drinking water in which residual chlorine could be detected.

\subsection{Experimental Design}

We implemented four randomly assigned and partially nested interventions (see Figures A1 for the design and A2 for the timeline). First, the Coupon intervention provided coupons that could be redeemed for a free bottle of WaterGuard, a point-of-use water treatment, at a nearby shop each month for 18 months (1,248 households received coupons and 1,225 were in the control group). In terms of the framework above, the rationale behind the coupon program is that, as shown in Dupas et al. (2016), it can reduce the gap between take $e_{m a r}$ and $u_{s e}$ mar by screening out non-users. The concern is that it may fail at encouraging usage among households who may have a high health return $b$ but either do not know it or for whom the hassle of redeeming the coupon is high, e.g. due to distance or forgetfulness. The second and third interventions attempt to palliate these. The second intervention is WASH promotion by CHWs. Three months after coupons were delivered, a random subset of the CHWs were trained on how to incorporate water hygiene discussions (in particular, chlorine treatment) into their monthly household visits (321 CHWs received training and 106 were in a control group). The third intervention is free home delivery: a random subset of the CHWs trained to promote chlorine were also instructed to make free deliveries of WaterGuard to all the households in their catchment area (106 CHWs and over 2,000 households, 458 of which are in our study sample). While free home delivery can help reduce exclusion errors, it may create a lot of 
inclusion errors, i.e. a huge gap between take $e_{m a r}$ and $u s e_{m a r}$ if distribution is not rationed. Our fourth intervention tests whether rationing home delivery helps improve targeting. After 6 months, a "rationing" treatment was introduced for CHWs in the home delivery group: a random subset were assigned to receive enough WaterGuard supply for only $40 \%$ or $60 \%$ of their households (the others continued receiving enough for $100 \%$ coverage).

\subsubsection{Treatment: Coupons for Free Chlorine (Household-level randomization)}

Households assigned to the coupon intervention received coupons that could be redeemed for a free bottle of WaterGuard at a local shop. Households could redeem one coupon per month for 18 months. Each coupon corresponded to one unique month (e.g. the coupon for March 2018 only be redeemed in March 2018). Coupons were attached to a calendar, the same calendar as that given to control households. and which many households displayed on their wall (see Appendix Figure A3). Each coupon was exchangeable for one 150mL bottle of WaterGuard, enough to treat a standard 20L jerrycan of water approximately 30 times. One bottle, if used correctly, is enough for roughly one month's supply of treated water (for drinking and cooking) for a family of 5 .

Shop owners were recruited to be part of the WaterGuard distribution network from whom households could redeem coupons. We recruited shops in all of the trading centers in our study area so that all households could redeem coupons at their closest trading center. The average distance to the closest shop where households could redeem coupons was $2.1 \mathrm{~km}$ (median $1.85 \mathrm{~km})$. Shop owners received an initial WaterGuard supply and were restocked each month as needed (no shop ever ran out). All participating shop owners received a monthly stipend of MKW 10,000 (roughly USD13) and signed a contract agreeing to 1) accept coupons in exchange for WaterGuard with no additional fee, 2) only accept coupons in the month for which the coupon was specified, 3) record each coupon redemption with a coupon serial number in a record book, 4) retain the coupon until collected by the study team, and 5) pay the cost of any missing bottles not recorded in the record book (approximately USD0.50). This generated highly reliable data on coupon redemption for each household. 


\subsubsection{Treatment: CHWs as WASH Promoters (CHW-level randomization)}

PIH trained a random subset of CHWs on how to promote safe drinking water practices: how to talk to households about the importance of water treatment, with emphasis on chlorine use, in addition to other aspects of Water, Sanitation, and Hygiene (e.g., proper boiling methods, handwashing, sanitation of cooking areas and materials and the safe storage of food). Trained CHWs were instructed to incorporate chlorine promotion into their monthly household visits. Therefore, households in the catchment area of a CHW assigned to this arm were expected to receive promotion of chlorine from their $\mathrm{CHW}$ at their home each month.

\subsubsection{Treatment: Home Delivery of Free Chlorine by CHWs (CHW-level ran- domization)}

A random subset of CHWs were trained by $\mathrm{PIH}$ on how to promote chlorine usage as above, but also assigned to deliver chlorine directly to households on a monthly basis. These CHWs received a stock of $150 \mathrm{ml}$ chlorine bottles at routine staff meetings held monthly. They received exactly one bottle per month per household in their catchment area and were instructed to deliver one bottle per household. Therefore, each household with a CHW in the home delivery group was expected to receive a free home delivery of chlorine each month. (Whether this happened is an outcome of interest and will be discussed in section 5.) Other materials, such as buckets for water, were not provided. As shown in Figure A1, this group did not overlap with the coupon arm: Households receiving some form of chlorine either received coupons or home delivery, but never both. In addition, there was no home delivery without WASH promotion (e.g. there is no home-delivery equivalent to the coupons-only arm).

\subsubsection{Rationing Intervention (CHW-level randomization)}

Eight months after the start of the home delivery treatment, $2 / 3$ of the CHWs in that arm were selected for the "rationing" intervention. Starting in February 2019, they were given enough WaterGuard for only a subset of their households (1/3 of all home delivery CHWs received enough for $60 \%$ of households and $1 / 3$ received enough for $40 \%$; the remaining $1 / 3$ continued receiving enough for all households). CHWs were told that data from household surveys suggested that only a minority of households used chlorine, therefore rationing was a 
wastage-reduction measure, and they were expected to use their local knowledge and discretion to decide whom to give the bottles to. They were explicitly asked to try to target households most likely to put the chlorine solution to good use.

\subsubsection{Randomization Procedures}

CHWs were randomly assigned to stay in the status quo (25\%), WASH Training (50\%), and WASH Training + Home Delivery (25\%), stratifying on gender.

In Neno, Households whose CHWs had not been assigned to home delivery were randomly assigned to receive coupons or no coupons, stratifying on cluster. In Mwanza, households were assigned randomly to receive coupons or no coupons, stratifying on geographic areas used for sampling.

\subsection{Data}

\subsubsection{Baseline Survey}

The baseline survey conducted in March-April 2018 measured basic household demographics and socio-economic status, as well as self-reported water quality and treatment, and child health. We also tested for the presence of chlorine in the drinking water using a chlorinometer.

All households received the same calendar at the end of the interview. For households in the Coupons treatment, 18 monthly coupons were attached to the calendar, and they received an explanation for how to redeem the coupons. Treatment status was blinded to both the household and the surveyor until the end of the interview.

\subsubsection{Rolling Follow-up Survey}

In order to trace usage over time, we conducted follow-up surveys on a rolling basis. Specifically, we visited around 260 households every month. We aimed to visit each household twice. The first round of visits started in May 2018 and was completed by mid-December 2018. The second round started immediately following the completion of the first round in mid-December 2018 and ended in June 2019. The order of the visits was random, but stratified by cluster. Households that were determined to have moved away permanently as of the first visit were not 
sampled again. From our baseline sample of 2,313 households, we completed follow-up surveys for 2,105 households in the first round and 1,731 during the second round. The attrition in round 2 was in part due to torrential floods that cut out access to entire sections of the sample area for a few months. Because we had some funds left, we randomly selected 90 households to receive a third visit in July 2019, of these 73 were surveyed.) See section 5.5.1 for an assessment of the potential bias resulting from attrition.

\subsubsection{Coupon Redemption Data}

Redeemed coupons were collected from enrolled shops on a monthly basis starting in March 2018 and the numbers scanned. Households for whom a coupon stub for a given month was not present at the shop are considered to have not redeemed the coupon.

\subsubsection{CHW Survey}

We completed two rounds of surveys with CHWs in Neno District. The first round of surveys was conducted prior to the CHW-level interventions. It collected data on CHW demographics, their roles outside the CHW program, and their beliefs with respect to community challenges. This survey was self-administered during routine area-level monthly staff meetings: An enumerator attended the meeting and explained each question to CHWs that were present, and they filled in the questionnaire on their own. This was completed in November 2017.

A second round of CHW surveys was completed in June 2019. The survey elicited CHW's views on the intervention and asked about household attitudes towards WaterGuard and the CHW's WaterGuard distribution activities (if applicable).

\subsection{Outcomes of Interest}

\subsubsection{Chlorine Usage: Chlorine Tests}

Household chlorine use was measured using colorimetric tests. Respondents were asked to provide a cup of water from their drinking water reserve, and enumerators added reagent powder which turned a shade of pink if residual chlorine was present. Enumerators compared this shade against a provided color wheel to determine the concentration of chlorine in the water. Two 
tests were done: one with free chlorine, which measures the concentration of available chlorine (i.e. whether there is any chlorine left in the water to keep it safe), and one for residual measures (i.e. whether there are any byproducts of a chlorine reaction). The two measures differed in less than $1 \%$ of the time so the study results are identical across the two measures, and for simplicity we focus on just one for the analysis (residual chlorine). Households with a non-zero concentration of residual chlorine are considered to have treated their water. Households with no drinking water reserve at the time of the visit are coded as not having treated water (158 observations $(5 \%$ of total $)) .^{9}$

\subsubsection{Child Health}

We measured three common illnesses in children that can be caused by consumption of contaminated water: diarrhea, fever and vomiting. Our enumerators asked caretakers about illness in all of their children under-10 years old that occurred within the last four weeks. We analyze the health data at the child level. We create a dummy for "any illness", and also consider the total number of illnesses experienced. We show the results for each illness separately, as well as for different recall periods and different age groups, in the appendix. We discuss potential reporting bias, in particular the risk of experimenter demand effect, in section 5.5.2.

We also measured coughing. Water-borne pathogens common in our setting do not have coughing as a symptom, but coughing is a symptom of indoor air pollution, which is high in rural Malawi, where wood burning is the primary source of cooking fuel (91\% of households in our sample). There are two potential indirect channels through which water treatment can reduce indoor air pollution exposure: by reducing the need to boil water, and by reducing illness spells during which children stay indoors. We discuss impacts on coughing in section 5.5.2.

\subsection{Estimation Strategy}

We provide results in both graphical and regression formats. The graphics show the raw data month by month - a nice feature of our rolling follow-up survey is that we can trace out impacts over time. This also allows us to study how treatment effects vary with seasons. The regression

\footnotetext{
${ }^{9}$ Table B1 shows that results are similar when we exclude these households rather than coding them as no treated water.
} 
results are based on the following specification:

$$
\begin{gathered}
y_{i t}=\beta+\theta C O U P O N_{i}+\alpha W A S H_{i t}+\delta\left(W A S H_{i t} \times C O U P O N_{i}\right)+\sigma M W A N Z A_{i}+ \\
\rho\left(M W A N Z A_{i} \times C O U P O N_{i}\right)+\nu D E L I V_{i t}+\psi\left(D E L I V_{i t} \times R A T I O N_{i t}\right)+\gamma_{t}+\epsilon_{i t}
\end{gathered}
$$

where $y$ represents the (follow-up) outcome for individual $i$ in quarter $t$ of the study, COUPON is an indicator for whether the individual is in the coupon group, $W A S H$ is an indicator for whether the individual's CHW has received the WASH promotion intervention (this turns from 0 to 1 in June 2018 for CHWs sampled for training), $M W A N Z A$ is an indicator for whether a household resides in Mwanza district, and DELIV is an indicator for whether the household's CHW was assigned to the home delivery arm (this also turns on in June 2018 for households with a CHW assigned to home delivery). RATION is an indicator for whether the household's CHW was assigned to one of the two rationing arms (this turns on in February 2019 for households with a CHW assigned to rationing). ${ }^{10}$ Finally, $\gamma_{t}$ is a vector of indicators for each quarter of the study (i.e., quarter fixed effects). In this set up, $\theta$ represents the coupon treatment effect in Neno district for the households who did not have a CHW assigned to the WASH promotion or delivery arm, $\alpha$ represents the effect of WASH promotion training in absence of the coupon intervention, $\delta$ represents the additional effect of the coupon intervention when the CHW is also assigned to incorporate WASH promotion, $\sigma$ is the effect of living in Mwanza relative to Neno, $\rho$ is the additional effect of the coupon intervention in Mwanza, $\nu$ is the effect of the home delivery intervention, and $\psi$ is the effect of the rationing intervention relative to home delivery without rationing. Our main analysis pools all post intervention time periods and excludes the baseline, since not all outcomes of interest were measured at baseline. Analyses controlling for baseline characteristics, including baseline values of the outcome of interest when available, yield identical results and are shown in Appendix Table B2. We cluster standard errors at the CHW level in all analyses because the WASH promotion and home delivery interventions were both assigned at the CHW level in Neno. ${ }^{11}$ This produces conservative standard errors for the coupon treatment effect, since the coupons were randomized at the household level.

\footnotetext{
${ }^{10}$ In the main specification (Table 2) we pool the rationing arms, but we study the impacts of rationing levels separately in a specification focused on the Home Delivery group in Table 5.

${ }^{11}$ In Mwanza clusters are households since there are no CHW program and the only intervention (coupons) was randomized at the household level.
} 


\section{7 $\quad$ Balance between study arms at baseline}

We estimate equation 1 using data from the baseline survey to assess balance on our main outcomes prior to the start of the interventions (Table A2). This set-up allows for easy comparison with our main coefficients. We find some slight imbalance on one of our two primary outcomes: presence of chlorine in the water. Specifically, Neno households assigned to the coupon arm without WASH promotion were 3.7 percentage points less likely to use chlorine at baseline than control households. This difference is statistically significant but economically small, and will be dwarfed by our estimates of the coupon treatment effects below, so it does not affect our ability to estimate causal impacts, and controlling for baseline levels in the analysis do not change the results (Table B2). Note that households assigned to coupons with WASH are not different than control households $(-0.037-0.027+0.066=0.02)$. Also note that when pooling all arms that received coupons, the coefficient on coupon assignment at baseline was trivial (0.4 percentage points). For all other outcomes, we do not see any imbalance. In particular, study arm assignment is not associated with the probability of a $\mathrm{CHW}$ visit in the 4-weeks prior to the baseline survey or with child health.

\section{Results}

\subsection{Impacts on Chlorine Usage and Child Health}

Table 2 shows the impacts on our primary outcomes of interest: whether we could detect chlorine in the household's drinking water during unannounced follow-up visits, and child health. This table also includes results for self-reported WaterGuard (likely an upper bound) and whether the household gave any WaterGuard away (spillover).

Both subsidy interventions had large impacts on water treatment rates. When pooling all arms that received coupons, the coupon intervention increased the likelihood of a positive chlorine test by 26.6 percentage points (see "Pooled Coupon Effect" at the bottom of the table), while the home delivery intervention increased it by 19.5 percentage points. These are vast improvements in chlorine use compared to the control group (only $4 \%$ of the control households had a positive chlorine test at follow-up). 
These large increases in water treatment rates from the subsidies led to significant improvements in child health as reported by the caretakers: the likelihood of a child under 10 experiencing any of the three illnesses in the past month decreased by 9.2 percentage points (pooled effect) in the coupon group (from a base of 43\%). The number of illnesses decreases by 0.15 from a base of 0.61 , a $24 \%$ reduction. The impact of the home delivery intervention is somewhat lower but cannot be distinguished statistically from the coupon effect. Overall, the magnitude of the effects on chlorine usage and child health are consistent across the two interventions, with the coupon intervention having a greater impact on both outcomes.

Figure 1 shows the percent change illness by illness for coupon and home delivery interventions, based on the coefficient estimates shown in Table A3. The coupon intervention significantly reduced episodes of diarrhea (25.7\% reduction), fever $(24.3 \%$ reduction), and vomiting $(26.9 \%$ reduction). Child illness reductions from the home delivery intervention were of important magnitudes for all illnesses, but imprecisely estimated for individual illnesses. While there was a significant reduction in child illnesses in Neno, the effect in Mwanza, where child health was substantially worse on all categories at baseline (0.76 illnesses compared to 0.58 in Neno, see Table 2, column 5), was much larger. This was particularly true for diarrhea: there was a 7.5 percentage point reduction in Mwanza compared to a 1.9 percentage point reduction in Neno (Table A3).

Our main results use a 4-week recall for illnesses among children under 10. Table B3 shows that our results for diarrhea are robust to different recall periods (14 days and 7 days); if anything, the relative increase in the pooled coupon effect increases with shorter recall. (We cannot do this exercise for vomiting and fever because we did not collect information allowing for different recall periods for those.) Table B4 provides the results for children under 5. They are similar to results for under 10 .

\subsection{WASH Promotion through CHWs does not increase usage}

Although the two subsidy schemes (coupons and home delivery by CHW) generate a considerable increase in water treatment rates compared to baseline levels, the usage rate is far from universal, with only $30 \%$ of households using free chlorine at a given point in time. Can the usage rate be increased among subsidy beneficiaries through behavioral change communica- 
tion? In the terms of section 3, can $u s e_{m a r}$ be increased as much as take $_{\text {mar }}$ ? In contrast to our hypothesis going in, the presence of a CHW program does not seem to increase chlorine usage, even when CHWs are specifically trained on WASH promotion. Direct delivery by trained CHWs did not yield higher usage rates than the coupon scheme, and training CHWs on WASH did not influence overall chlorine usage in the coupon group: it did not affect coupon redemption (Figure 2), nor did it influence chlorine usage conditional on having redeemed the coupon. ${ }^{12}$ We also note that water treatment rates are not higher in Neno (where the CHW program operates) than Mwanza, be it without or with coupons (Table 2).

\subsection{Targeting}

Increasing the usage rate among takers appears difficult. This suggests that targeting subsidies to those most likely to use the product is important. The main rationale for a coupon scheme is that it can generate self-targeting: since there is essentially no use for a small diluted chlorine bottle outside of water treatment, and very little (if any) resale value, households that have no intention to use the product may not bother redeeming the coupon. Figures 2 and 3 show the patterns of results for the coupon intervention over time. We find substantial self-targeting: While coupon redemption decreased over time from $74 \%$ in month 1 to $35 \%$ in month 18 , chlorine usage as measured through the water tests holds steady at about $30 \%$ of coupon households for the duration of the study. This suggests that the drop in coupon redemption in the first few months is among people who had weaker preferences for chlorine use and that self-targeting improved over time. What's more, we find that the health effects are also very consistent across months, suggesting that the health return for self-selected marginal users under the coupon scheme $\left(b_{\text {mar }}\right)$ is high. This has promising implications for the efficiency of this program in the long run.

So far we have shown that about $30 \%$ of households that received coupons treat their water in a given month. However, it is unclear if it is the same $30 \%$ of households each month or if households treat some months but not others. This has implications for chlorine promotion programs, targeting chlorine to users, and for understanding chlorine preferences. We provide

\footnotetext{
${ }^{12}$ This is true regardless of the CHW's characteristics, which we assessed by interacting the WASH indicator with CHW age, education, number of households, hours spent on CHW activities, whether CHW is their primary source of income, and whether they were a senior CHW.
} 
insight into these issues by analyzing the distribution of number of coupons redeemed and by analyzing consistency in use across the waves of water testing.

Appendix figure A5 shows that there is bunching in the distribution of number of coupons redeemed around 0 and 1 (15\% of households) and around 16, 17 , and 18 (25\% of households). ${ }^{13}$ To assess consistency in chlorine use, Table 3 estimates treatment effects on whether households had a positive chlorine test at any of the two follow-up visits (column 1) and on whether they had a positive test at all follow-up visits (column 2). The pooled coupon effect shows that coupons increased the likelihood of having at least one positive chlorine test by 40.2 percentage points and of consistent chlorine use by 15.5 percentage points. Thus, more than half of the households that used chlorine as a result of the coupon intervention did not use it consistently, even in the WASH group. This could be because households only use chlorine at specific times - e.g. when a child is ill, or when the main water source is suddenly contaminated. Alternatively, this could be because other barriers such as lack of buckets, inconvenience or cognitive overload prevent people that would prefer to consistently use free chlorine from doing so. This is consistent with a recent experiment in Kenya, which shows that interventions that increase self-efficacy as well as the salience of chlorination can increase chlorine usage by $25 \%$ (Haushofer et al., 2019).

\subsubsection{Targeting on Health Returns: Heterogeneity by Baseline Water Quality}

Not all households need chlorine all the time to have clean water. At baseline, $71 \%$ of households report using a protected source (public tap, borehole with handpump, protected well, protected spring or private tap) and $50 \%$ believe that their water source is always safe. If they are correct, then the returns to using chlorine would be very low for such households. Table A4 zooms in on the control group, and examines the correlation between beliefs about water cleanliness and child illness. We find a strong negative correlation between whether the respondent "believes water is always safe" and child illness incidence, and likewise a strong negative correlation between "protected source" and child illness. This suggests that water sources for these households are truly less likely to be contaminated.

\footnotetext{
${ }^{13}$ Most households had the opportunity to redeem 18 coupons but about $10 \%$ did not receive their coupons until the first month had already passed. Also, some received their coupons towards the end of the first month and therefore might not have had time to redeem the first coupon.
} 
Ideally, the social planner would only target subsidized chlorine to households with contaminated water. We test for how well coupons and home delivery by CHWs target chlorine to households with contaminated water by examining whether baseline water cleanliness predicts chlorine use and child health at follow-up. Figure 5 shows that the coupon effect on chlorine use was significantly larger for households with an unprotected water source (35 percentage point increase compared to 22 percentage point increase; $\mathrm{p}<0.001)$. Correspondingly, the impacts on child health is also much larger for such households. These results imply a non-trivial degree of self-targeting based on perceived value of chlorine treatment.

In contrast, the effects of the home delivery treatment are not greater for households with worse water access at baseline. How come? Our data shows that CHWs appear to neglect such households relatively more than better endowed households. This is shown in Table 4, which tests whether households who have a less clean water source are those most likely to receive (if in the home delivery group) or take advantage of (if in the Coupon group) the free chlorine subsidy. We find that this is not the case for the home delivery treatment: CHWs visit those whose source is unprotected less regularly (column 1) and correspondingly deliver free chlorine bottles less often to such households (column 2). In contrast, self-targeting under the coupon program works well, with those with unprotected water being more likely to report they got free chlorine. The coupon redemption data (column 3) verifies this: households with an unprotected source redeem 19 percentage points (three) more coupons, on average, than those with a protected source. ${ }^{14}$ Column 3 also shows that households that were further from the shop where coupons could be redeemed were less likely to redeem coupons.

Why do CHWs fail to appropriately target households who have the greatest need for chlorine? This may be because such households are further away, hence the costs associated with doing a home visit are higher. Indeed, households whose distance to the trading center is greater than the median in the sample were significantly less likely to receive regular $\mathrm{CHW}$ visits, and the home delivery intervention did not change this (Column 4 of Table 4). Correspondingly, distance reduced the impact of the home delivery intervention on receipt of free WaterGuard (column 5). Strikingly, it did more so than in the coupon group. In other words, distance

\footnotetext{
${ }^{14}$ This is true even when we do not control for distance to the shop where the coupons could be redeemed. Average distance was significantly greater for households with an unprotected source: $2.3 \mathrm{~km}$ vs. $1.9 \mathrm{~km}$, a $400 \mathrm{~m}$ gap $(\mathrm{p}<0.001)$.
} 
impedes access to the subsidy more when the distance cost is paid by the delivery agent rather than the end-user.

\subsubsection{Targeting on Usage Cost: Heterogeneity by Baseline Taste Concerns}

Another dimension of heterogeneity is in households' distaste for chlorinated water. The minimum amount of chlorine necessary to purify water may not affect the taste of water, but dosing correctly can be difficult for non-standard water containers, so as households err on the side of caution, the actual quantities used to purify tend to give the water a chlorinated taste. At baseline, while $51 \%$ of respondents had ever used Waterguard, $45 \%$ of respondents mentioned that they think it gives water a bad taste, suggesting that this may be a serious barrier to adoption. Column 3 of Table 4 shows that households with such beliefs at baseline redeemed 8 percentage points (14\%) fewer coupons, suggesting self-targeting based on taste concerns. Figure A6 shows that the effects of the coupon scheme on chlorine use were smaller for households who believe WaterGuard alters the taste.

\subsubsection{Targeting through Ex-Post Sharing}

To the extent that subsidy recipients who do not care for chlorine give the chlorine away to other households, the overall targeting performance of the subsidy programs may be higher than what we observe when focusing on beneficiary households only. Coupon households are 15.6 percentage points more likely to report having ever given away WaterGuard to a neighbor than control households (Table 2). Because we provided coupons to less than $10 \%$ of households in a village, those spillovers are diffuse, however - chlorine usage in the control group was still very low at only $4 \%$ on average, not higher than at baseline. Home delivery households were not more likely to report giving WaterGuard away; this is possibly because their neighbors were already receiving WaterGuard from the CHW.

\subsubsection{Targeting by CHWs: Results from the Rationing Experiment}

Can CHWs be effective targeting agents when specifically asked to do so? Starting in February

2019, the rationing intervention randomly varied the amount of WaterGuard bottles CHWs had to distribute. This allows us to test whether CHWs, when they are in charge of identifying 
beneficiaries, can target free chlorine to households that use it.

Table 5 shows how the rationing arms compare to the $100 \%$ coverage arm on whether the households received a bottle in the last 4-weeks, positive chlorine tests, and any child illness. Columns 1-3 analyze each rationing arm separately and columns 4-6 pool them. This analysis only includes the 105 CHWs that were assigned to home delivery and fewer survey waves (261 households). Therefore, we have less power to detect effects than in our previous analyses.

Rationing supply of WaterGuard bottles was far more efficient than $100 \%$ coverage in terms of targeting users. Despite many fewer bottles being given to CHWs, rationing had small and insignificant effects on objectively measured WaterGuard usage among households (columns 2 and 5). This suggests that CHWs have private information about household's preferences for WaterGuard, and can effectively target bottles to households that will use them. Figure 4 plots the share of households that received WaterGuard and the share with positive chlorine tests by study month. By the last study month, the share of households that receive WaterGuard and the share that have treated water nearly converge in the rationing arms, whereas a large gap remains in the $100 \%$ coverage arm.

The estimates of the health effects under rationing are noisy due to the reduced sample size (fewer households over fewer months), but suggest that targeting by CHWs, while it is based on usage, may not be based on underlying health needs: the health effects under rationing cannot be distinguished from zero (Table 2 and Figure A4).

Overall, it seems clear from our results that absent a monitoring and/or incentive system, targeting through CHWs performs worse than self-targeting through coupon redemption. One prima facie puzzling result is that the self-targeting observed in the coupon scheme cannot be reproduced through the Home Delivery by CHW scheme. Why don't households who would go through the hassle of redeeming their coupons go through the hassle of heckling their CHW to visit them / give them chlorine? Why don't households who would not bother to redeem their coupon still accept the free bottle from the CHW? On the former, CHWs may not have informed households that they were entitled to a free bottle every month, reducing the pressure on themselves to make monthly visits, especially to far away households. Once they had to ration, CHWs may have asked households directly: "Will you use the chlorine? I need to 
know because I do not have enough for everyone." This could explain why targeting on usage improves under rationing, but it improved targeting only among household that CHWs cared to approach. On why households do not opt out of the free delivery unless explicitly encouraged to do so, the most likely explanation is that it is difficult to refuse a free health good from a health worker, likely due to social desirability bias.

\subsection{Why is chlorine promotion by CHWs not making a difference?}

CHWs employed by PIH have been working in Neno to support the primary care system for over 12 years and are a respected part of the health system in the district. In 2014-2015, CHWs were trained and deployed to identify pregnant women and escort them to prenatal care visits, and a synthetic control method study estimated that this led to an $18 \%$ increase in enrollment for prenatal care (Kachimanga et al., 2020). Based on this, PIH expected that WASH advice from CHWs would be followed, especially if it comes with the hand-delivery of a subsidized product. Why, then, did the WASH training not improve household usage of chlorine, even when combined with home delivery?

A first possible explanation is that CHWs have a large and growing amount of responsibilities and might not have the time to implement the intervention as designed. This could have led CHWs to forgo visiting some households. Moreover, during household visits, CHWs are tasked with a long checklist of items to complete, which could crowd out chlorine promotion during the visit. We test for these potential explanations by estimating equation (1) to predict the probability of receiving a CHW visit in the previous 4 weeks and for the probability of a CHW talking about chlorine during their last visit (Table 6, columns 1 and 2). Only 58.5\% of households received a home visit in the previous 4 weeks in the control group and there was no significant difference in the chlorine promotion arm (column 1). (The figure increases to $80 \%$ for any CHW visit in the last 2 months). Interestingly, the home delivery intervention increased CHW effort: home delivery led to a 12 percentage point (22\%) increase in households reporting a home visit from a CHW. This result suggests that the ability to deliver free goods may motivate CHWs to visit households more regularly than they would otherwise. This is consistent with the finding from Wagner et al. (2020) in Uganda, where CHWs asked to deliver free ORS kits to households did more home visits than when they could sell the treatment kits 
door-to-door and keep the revenue. Nevertheless, in our settting compliance with home delivery to all households was not perfect among CHWs, with only $58 \%$ of households receiving a home delivery from their CHW absent any rationing (Table 6).

Column 2 Table 6 shows that over a quarter of control households receive information about chlorine from CHW to start with, and the increase in exposure to chlorine promotion was much lower than expected in the WASH arm. Assignment to WASH training increased the likelihood of the CHW talking about chlorine during their previous visit by 13.5 percentage points from $25.7 \%$ in the control arm. But interestingly, this effect is no greater than the effect of coupons alone: households who received coupons were 11.4 percentage points more likely to have their non-trained CHW discuss WaterGuard with them during their last visit. It could be that since households in the coupon arm are more likely to have chlorine on hand, they bring up the topic themselves, or that the CHW reacts to the coupons on the wall calendar. Ultimately, the WASH intervention did not lead to a significant change in attitudes about the need to treat water with chlorine (column 3), beliefs about the effectiveness of WaterGuard at preventing illness (columns 5 and 6), or knowledge of correct use (column 8). It is also possible that informing households that their water is not safe encourages households to adopt other strategies to clean their water besides chlorine, such as boiling. Column 4 does not support this hypothesis, showing that households in the WASH arm were no more likely to boil their water.

\subsection{Potential Threats to Internal Validity}

\subsubsection{Attrition}

About $6 \%$ of households assessed at baseline received no follow-up visits and $22 \%$ received only one follow-up visit. ${ }^{15}$ Table A5 regresses the number of follow-up visits per household on their treatment assignment. This table shows that the probability of attrition was similar across study arms. However, it is possible that households who missed a follow-up visit had different characteristics across study arms. Table A6 shows baseline characteristics of attriters (the 608 households that had less than two follow-up visits). Differences among attriters between

\footnotetext{
${ }^{15}$ The attrition in round 2 was in part due to torrential floods that cut out access to entire sections of the sample area for a few months.
} 
arms were small and mostly insignificant. Attriters in the coupon arm had less child illness at baseline, which implies, if anything, our child health results would be biased towards zero. However, it is still possible attriters were different on unobservable characteristics that are correlated with our main outcomes. To bound the bias that could result from this, we include attriters in our main regression models and assume that attriters from coupon and home delivery assigned households had bad outcomes (no treated water and child illness) and attriters from control households had good outcomes (treated water and no child illness). ${ }^{16}$ Table A7 shows that under these extreme assumptions, we still see a statistically significant 6 percentage point increase (27\% increase) in chlorine use, but effects on child illness become very imprecise.

\subsubsection{Reporting Bias for Health Outcomes}

While our measure of chlorine usage is objectively measured through water tests, our measures for health outcomes are self-reports by caregivers. Since study participants could obviously not be blinded to their treatment status, one could be concerned that the impacts we observe are due to experimenter demand effects. Wolf et al. (2018) suggest that non-blinding could lead researchers to overestimate diarrhea reductions. In this section, we discuss various pieces of evidence to help allay such concerns. First, we note that the health effects for the coupons persist over time (as shown in Figure 3), while experimenter demand effects are typically short-lived. Second, as shown in Figure 5, we see smaller impacts on child health for those reporting having a protected source of water, as expected. Third, we observe spillover effects on respiratory health (see column 4 of Table A3). Specifically, the incidence of coughing, very high in our sample due to indoor air pollution (45\% of children under 10 experienced coughing in the previous 4 weeks in the control group), reduces by $15 \%$. There is no reason to think that experimenter demand effects would carry through to respiratory health, an illness clearly not targeted by the water treatment subsidy program. The decrease in coughing is consistent with the observed decrease in water boiling (Table 6), as well as the fact that children spend more time outdoors when they are not sick with diarrhea or fever. Fourth, we see larger health impacts in Mwanza than Neno, consistent with greater coupon usage in Mwanza, but there is no reason why experimenter demand effects would be greater in Mwanza. Finally, the treatment that may have been the most

\footnotetext{
${ }^{16}$ We exclude the WASH treatment from this exercise for simplicity
} 
likely to generate social desirability bias is the WASH treatment, since it explicitly encourage households to use chlorine. Indeed, in Table 2, we see that the WASH treatment shows a significant effect in chlorine usage when self-reported (column 2) that is not validated with the objective chlorine test data (column 1). Yet households in the WASH treatment do not report fewer illnesses (columns 4 and 5), suggesting that if experimenter demand effects may have been at play in self-reporting of chlorine usage (the rationale to do objective water tests), they do not seem to carry through to caretaker reported health outcomes. Overall, while we acknowledge that having objective measures of child health would have been a great addition to this study had it been possible, we take our estimated effects on caregiver-reported health outcomes as strongly suggestive.

\section{Cost-effectiveness}

In order to estimate the cost-effectiveness of the coupon and home delivery interventions, we use administrative data on number of bottles distributed and distribution costs combined with our treatment effect estimates. We restrict our analysis to the last 12 months of the interventions (September 2018 onward) to get closer to steady-state cost and effectiveness estimates. We estimate home delivery cost-effectiveness separately for the versions with $100 \%, 60 \%$, and $40 \%$ coverage.

We calculate the cost per additional 30 household-days with treated drinking water and the cost per child illness averted. The two analyses call for different time horizons because households with treated water is reflective of one point in time, whereas child illnesses averted add up over time. Thus, we used monthly costs and effectiveness (averaged over all 12 months) when estimating cost per additional 30 household-days with treated drinking water, and total costs and effectiveness for the full 12 months when estimating the cost per child illness averted. We use household-days because our chlorine tests only verify whether a household had chlorine tests on a given day. Thus, each positive test is 1 household-day with treated water. We consider a child as having illness if their caretaker report that they had diarrhea, fever, or vomiting in the

previous 4 weeks. Treatment effects for illnesses averted are from the specification in equation 1, restricting to data collected after September 1, 2018. 
Cost estimates are based on the number of WaterGuard bottles distributed and the wholesale price (USD 0.45 per bottle). We use coupon redemption data to identify the number of bottles redeemed in the coupon arm, and PIH administrative records to identify the number of bottles handed out to CHWs in the home delivery arms. We also include the monthly cost of distribution to shops in the coupon arm and monthly cost of distribution to CHWs in the home delivery arm. ${ }^{17}$ The monthly distribution costs to shops are likely an upper bound because a scaled up version could deliver to shops less frequently (e.g. every 3 months). CHWs, however, would have difficulty transporting and storing more than one month's supply.

Table 7 shows the cost-effectiveness estimates. Panel A shows that coupons were far more costeffective than home delivery with $100 \%$ coverage in terms of increasing chlorine use, and that rationing helped improve the cost-effectiveness of home delivery. Among the 872 households that received coupons, an average of 419 coupons were redeemed per month, costing about $\$ 258$ ( $\$ 0.29$ per household). This led to 6,804 additional household-days with treated drinking water. ${ }^{18}$ This gives a cost effectiveness ratio (CER) of $\$ 01.13$ per 30 households-days with treated water. We distributed more bottles in the home delivery arms because the intervention applied to all households in the CHW's catchment area (close to 1,000 households in each arm), whereas coupons only applied to those we directly enrolled in the study during the baseline visit. The 100\% coverage arm was the most expensive and the least cost-effective. While the $40 \%$ rationing arm has the lowest CER, the CERs for both the rationing arms are very noisy, because we only implemented these interventions for six months rather than 18 .

Panel B shows that the coupons intervention cost $\$ 2.69$ to avert one child illness. The home delivery arms were also relatively cost-effective in terms of averting illness, but less so than coupons. The home delivery with $100 \%$ coverage intervention cost nearly twice as much per household as the coupon intervention and is less cost-effective at $\$ 12.54$ per illness averted. ${ }^{19}$ Coupons cost $\$ 6.40$ per diarrhea case averted vs. $\$ 24$ for home delivery (Table A8).

\footnotetext{
${ }^{17}$ It cost the program roughly $\$ 25$ per shop to make the deliveries each month and each shop covers about 313 households ( $\$ 0.08$ per household). Distribution to CHWs is more centralized since CHWs meet monthly at the health centre, and hence cheaper at $\$ 0.02$ per household per month.

${ }^{18}$ On any given day $227(0.26 \times 872)$ additional households had a positive test. Over the entire month this is 6,804 household days.

${ }^{19}$ We did not implement any of the home delivery arms for an entire 12 months because we started the $100 \%$ coverage intervention 3 months after baseline and the rationing intervention 10 months after baseline. Therefore we extrapolated 12 month costs using the monthly costs, which are fixed.
} 


\section{Conclusion}

Governments of low- and middle-income countries spend relatively large sums on water and sanitation subsidies: between 1.5 and $2 \%$ of GDP according to a recent World Bank report (Andres et al., 2019). The incidence of this spending has recently been questioned, however. Careful analysis of the spending in 10 countries suggests that $56 \%$ of subsidies end up in the pockets of the richest $20 \%$ of households, while only $6 \%$ of subsidies find their way to the poorest $20 \%$ (Andres et al., 2019). The main cause of such poor targeting is that existing subsidies typically target piped water systems, which are largely nonexistent in rural areas, where the majority of the poor live. Even when poor households are in areas with access, most of them are unable to afford a connection to the network (Devoto et al., 2012). Identifying targeting mechanisms that can direct resources to the poor while excluding the more well-off is a key priority for governments.

This paper shows that one possible such mechanism-free water treatment solution made available monthly to households with young children conditional on them coming to pick it up from a central place-can effectively target subsidies to poor households whose drinking water is

unclean. Such a program is extremely cost-effective and substantially reduces the incidence of water-borne diseases in low density, unconnected rural areas, with no decrease in impact over an 18-month period. It is more cost-effective than relying on existing networks of CHWs. CHWs are worse at targeting subsidies to households that need them the most than self-targeting. What's more, their ability to nudge more households to treat water appears very limited, and asking them to do so may come at the expense of other tasks they have to do.

As far as we know, no country to date routinely provides free water treatment product to parents of young children through rural clinics. This situation reminds us of the situation regarding malaria prevention in the early 2000s (Cohen and Dupas, 2010). Since then, free distribution of insecticide-treated bednets to pregnant women and young children has been adopted by over thirty countries, and malaria incidence has considerably reduced as a result (World Health Organization, 2019). Our results suggest that adopting a similar policy for point-of-use water treatment would bring countries closer to achieving the goal of universal access to safe drinking water set forth by the 2030 Sustainable Development Agenda. 


\section{References}

Anderson, M. L. (2008). Multiple inference and gender differences in the effects of early intervention: A reevaluation of the abecedarian, perry preschool, and early training projects. Journal of the American Statistical Association, 103(484):1481-1495.

Andres, L. A., Thibert, M., Lombana Cordoba, C., Danilenko, A. V., Joseph, G., and Borja-Vega, C. (2019). Doing More with Less. World Bank. Available at https://elibrary.worldbank.org/doi/pdf/10.1596/32277.

Arnold, B. F. and Colford, J. M. (2007). Treating water with chlorine at point-of-use to improve water quality and reduce child diarrhea in developing countries: A systematic review and meta-analysis. The American Journal of Tropical Medicine and Hygiene, 76(2):354-364.

Banerjee, A. V., Duflo, E., Glennerster, R., and Kothari, D. (2010). Improving immunisation coverage in rural india: clustered randomised controlled evaluation of immunisation campaigns with and without incentives. $B M J, 340$.

Björkman-Nyqvist, M., Guariso, A., Svensson, J., and Yanagizawa-Drott, D. (2019). Reducing child mortality in the last mile: Experimental evidence on community health promoters in uganda. American Economic Journal: Applied Economics, 11(3):155-92.

Clasen, T., Schmidt, W.-P., Rabie, T., Roberts, I., and Cairncross, S. (2007). Interventions to improve water quality for preventing diarrhoea: systematic review and meta-analysis. $B m j$, 334(7597):782.

Cohen, J. and Dupas, P. (2010). Free Distribution or Cost-Sharing? Evidence from a Randomized Malaria Prevention Experiment. The Quarterly Journal of Economics, 125(1):1-45.

Devoto, F., Duflo, E., Dupas, P., Parienté, W., and Pons, V. (2012). Happiness on tap: Piped water adoption in urban morocco. American Economic Journal: Economic Policy, 4(4):6899.

Dizon-Ross, R., Dupas, P., and Robinson, J. (2017). Governance and the effectiveness of public health subsidies: Evidence from ghana, kenya and uganda. Journal of Public Economics, 156:150 - 169 . 
Duflo, E., Dupas, P., Ginn, T., Grace, M. B., Baraza, M., Pouliquen, V., and Sharma, V. (2019). Hiv prevention among youth: A randomized controlled trial of voluntary counseling and testing for hiv and male condom distribution in rural kenya. PLoS One, 14(7).

Dupas, P., Hoffmann, V., Kremer, M., and Zwane, A. P. (2016). Targeting health subsidies through a nonprice mechanism: A randomized controlled trial in kenya. Science, 353(6302):889-895.

Dupas, P. and Miguel, E. (2017). Chapter 1 - impacts and determinants of health levels in low-income countries. In Banerjee, A. V. and Duflo, E., editors, Handbook of Economic Field Experiments, volume 2 of Handbook of Economic Field Experiments, pages 3 - 93. North-Holland.

Fewtrell, L., Kaufmann, R. B., Kay, D., Enanoria, W., Haller, L., and Colford, J. M. (2005). Water, sanitation, and hygiene interventions to reduce diarrhoea in less developed countries: a systematic review and meta-analysis. The Lancet Infectious Diseases, 5(1):42 - 52 .

Haushofer, J., John, A., and Orkin, K. (2019). Can Simple Psychological Interventions Increase Preventive Health Investment? NBER Working Papers 25731, National Bureau of Economic Research, Inc.

Jamison, D. T., Gelband, H., Horton, S., Jha, P., Laxminarayan, R., Mock, C. N., and Nugen, R., editors (2018). Disease Control Priorities: Improving Health and Reducing Poverty, volume Disease Control Priorities, Vol. 9. The World Bank, 3rd edition edition.

Kachimanga, C., Dunbar, E. L., Watson, S., Cundale, K., Makungwa, H., Wroe, E. B., Malindi, C., Nazimera, L., Palazuelos, D., Drake, J., et al. (2020). Increasing utilisation of perinatal services: estimating the impact of community health worker program in neno, malawi. $B M C$ Pregnancy and Childbirth, 20(1):1-10.

Kremer, M., Miguel, E., Mullainathan, S., Null, C., and Zwane, A. P. (2011). Social engineering: Evidence from a suite of take-up experiments in kenya. Unpublished Working Paper.

Mekonnen, Z., Gelaye, K., Were, M., Gashu, K., and Tilahun, B. (2019). Effect of mobile text message reminders on routine childhood vaccination: a systematic review and meta-analysis. Systematic Reviews, 8. 
Milkman, K. L., Beshears, J., Choi, J. J., Laibson, D., and Madrian, B. C. (2011). Using implementation intentions prompts to enhance influenza vaccination rates. Proceedings of the National Academy of Sciences, 108(26):10415-10420.

NSO and ICF (2017). Malawi demographic and health survey 2015-16. Technical report, National Statistical Office Malawi and ICF, Zomba, Malawi. Available at http://dhsprogram.com/pubs/pdf/FR319/FR319.pdf.

Wagner, Z., Asiimwe, J. B., Dow, W. H., and Levine, D. I. (2019). The role of price and convenience in use of oral rehydration salts to treat child diarrhea: A cluster randomized trial in uganda. PLoS medicine, 16(1).

Wagner, Z., Asiimwe, J. B., and Levine, D. I. (2020). When financial incentives backfire: Evidence from a community health worker experiment in uganda. Journal of Development Economics, 144:102437.

Wolf, J., Hunter, P. R., Freeman, M. C., Cumming, O., Clasen, T., Bartram, J., Higgins, J. P., Johnston, R., Medlicott, K., Boisson, S., et al. (2018). Impact of drinking water, sanitation and handwashing with soap on childhood diarrhoeal disease: updated meta-analysis and meta-regression. Tropical medicine $\& 3$ international health, 23(5):508-525.

World Health Organization (2019). World malaria report 2019. resreport, WHO. Available at https://www.who.int/publications-detail/world-malaria-report-2019. 


\section{Figures and Tables}

Figure 1: Percent change in child illness

(a) Coupons

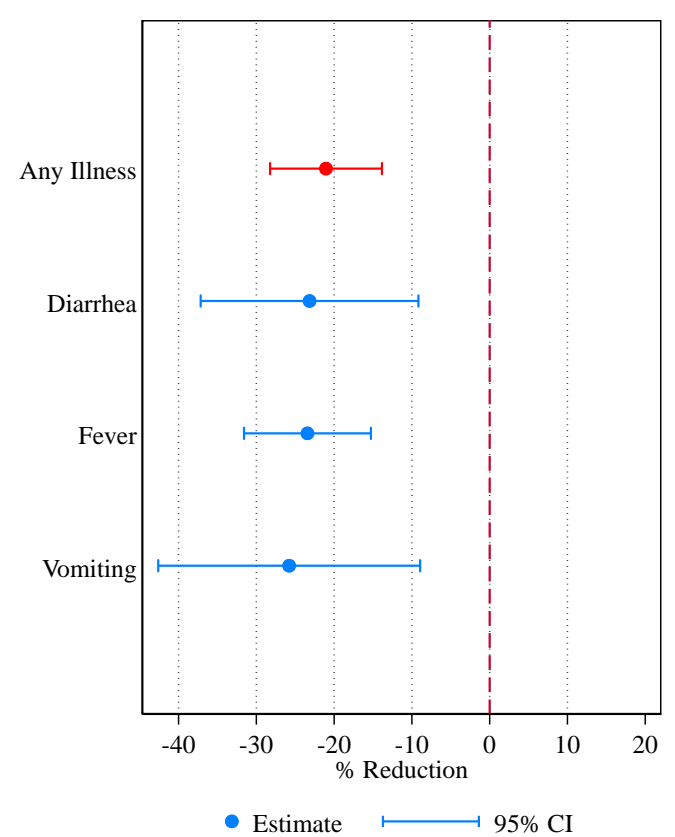

(b) Home Delivery

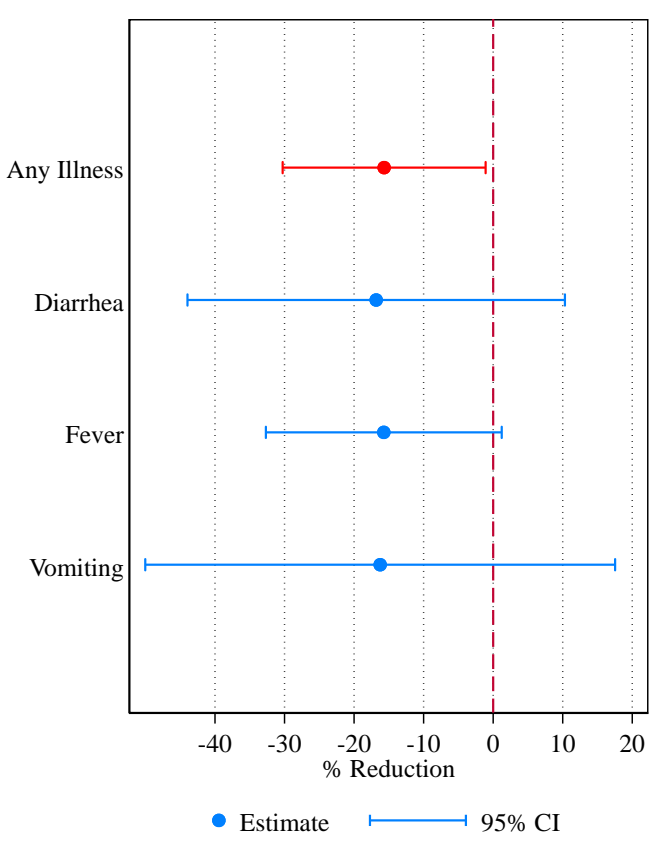

Notes: Percent changes are from regressions reported in Tables 2 and A3. We used the margins command in Stata to estimate the share of children that had each illness with and without the intervention and each point represents the percent difference in the intervention group relative to the control group. $95 \%$ confidence intervals are based on the upper and lower ends of the confidence interval of the difference divided by the level in the control group. 
Figure 2: Coupon redemption and verified usage, over time

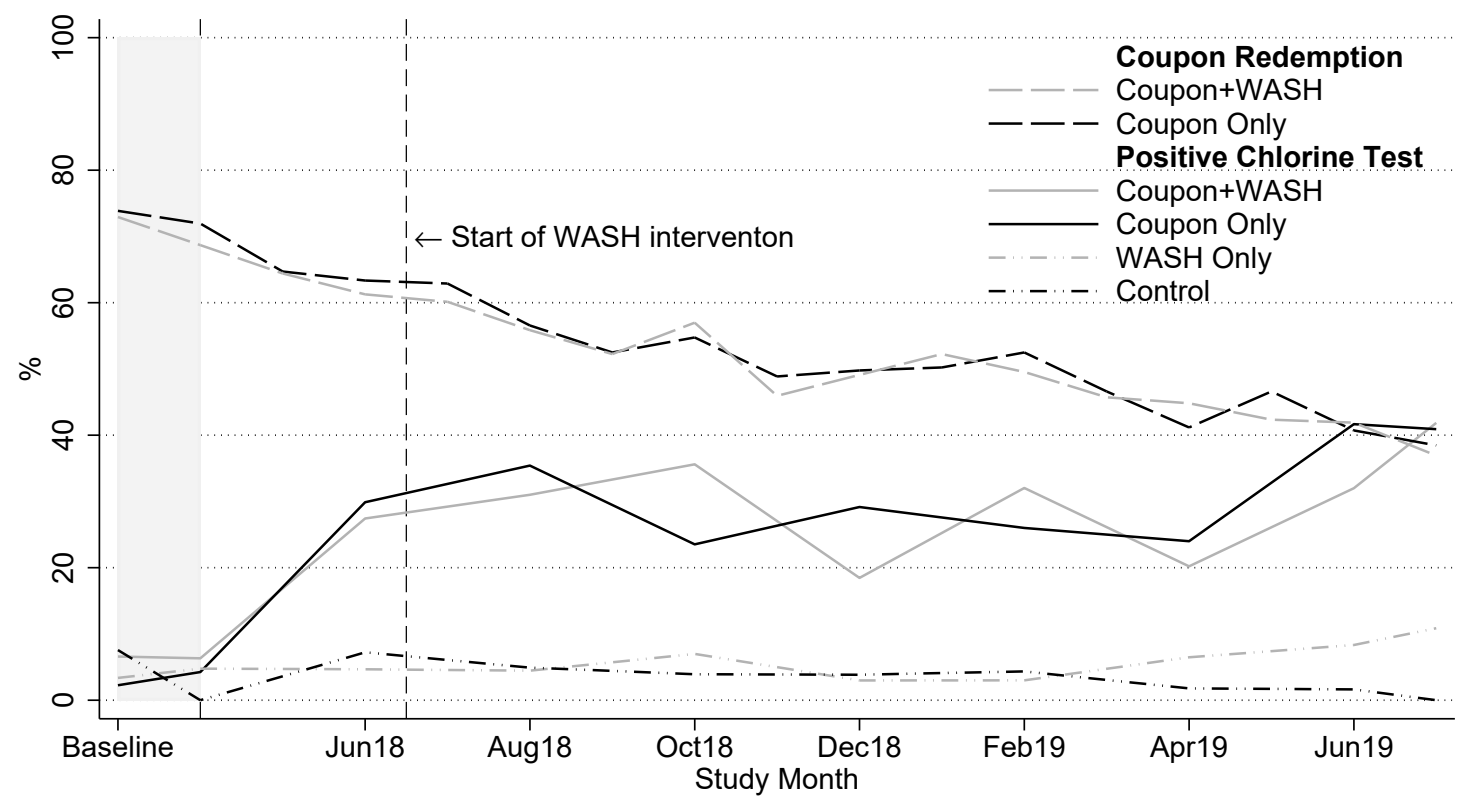

Notes: Positive chlorine test results are pooled in 2-month bins to reduce noise (e.g., May and June 2018 are pooled). An average of 267 households were surveyed each month. All water tests from the baseline period (March and April 2018) were conducted before the households received coupons. However, coupon redemption started for some households before all households received coupons.

Figure 3: Share of children with illness in previous 4 weeks, over time

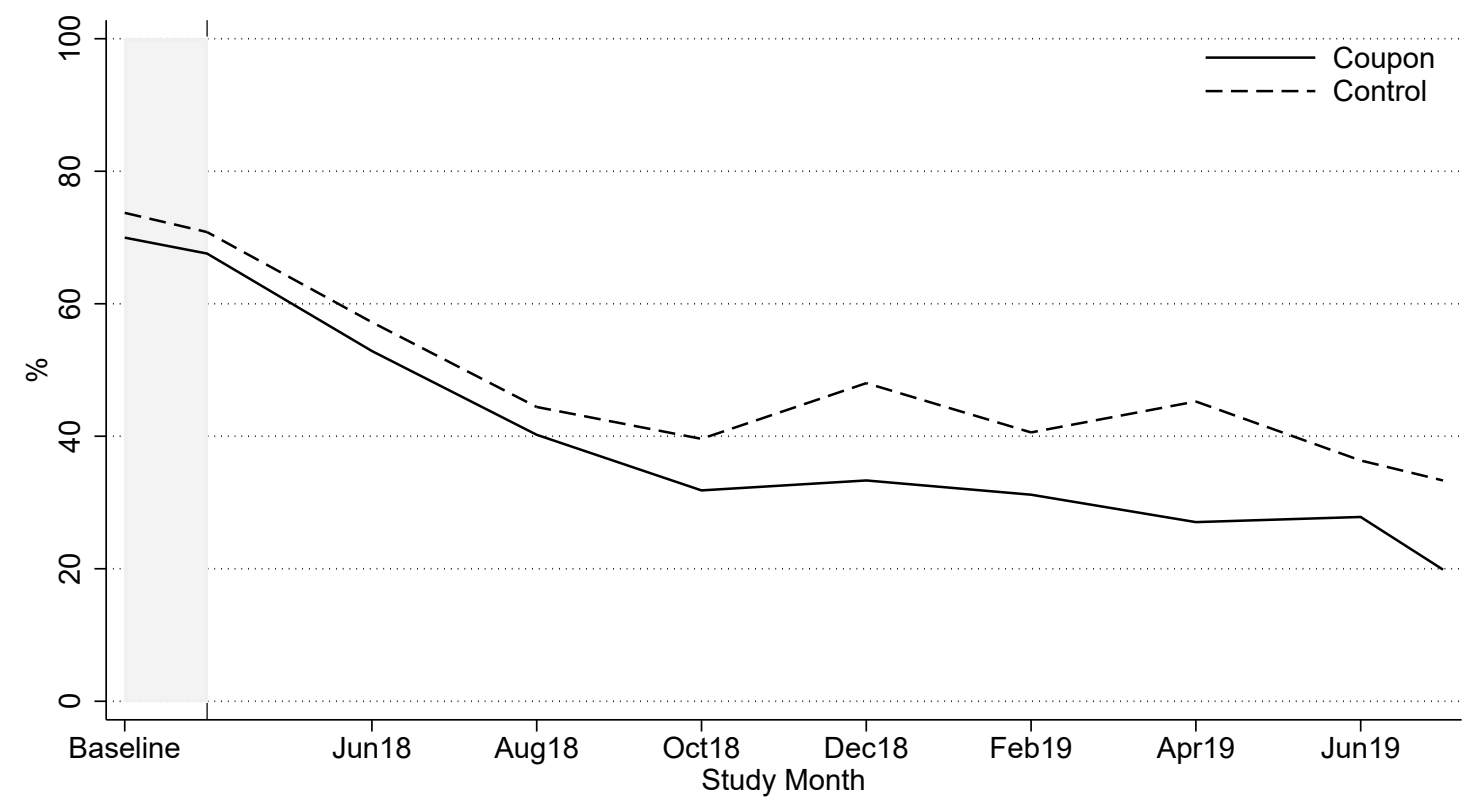

Notes: Lines plot share of children under 10 years old from surveyed households that had at least one illness among diarrhea, fever, and vomiting. Results pooled in 2-month bins to reduce noise. Each group pools respondents from the Non-WASH and WASH sub-groups. 
Figure 4: Home Delivery Group: Receipt of free WaterGuard and verified use by rationing assignment

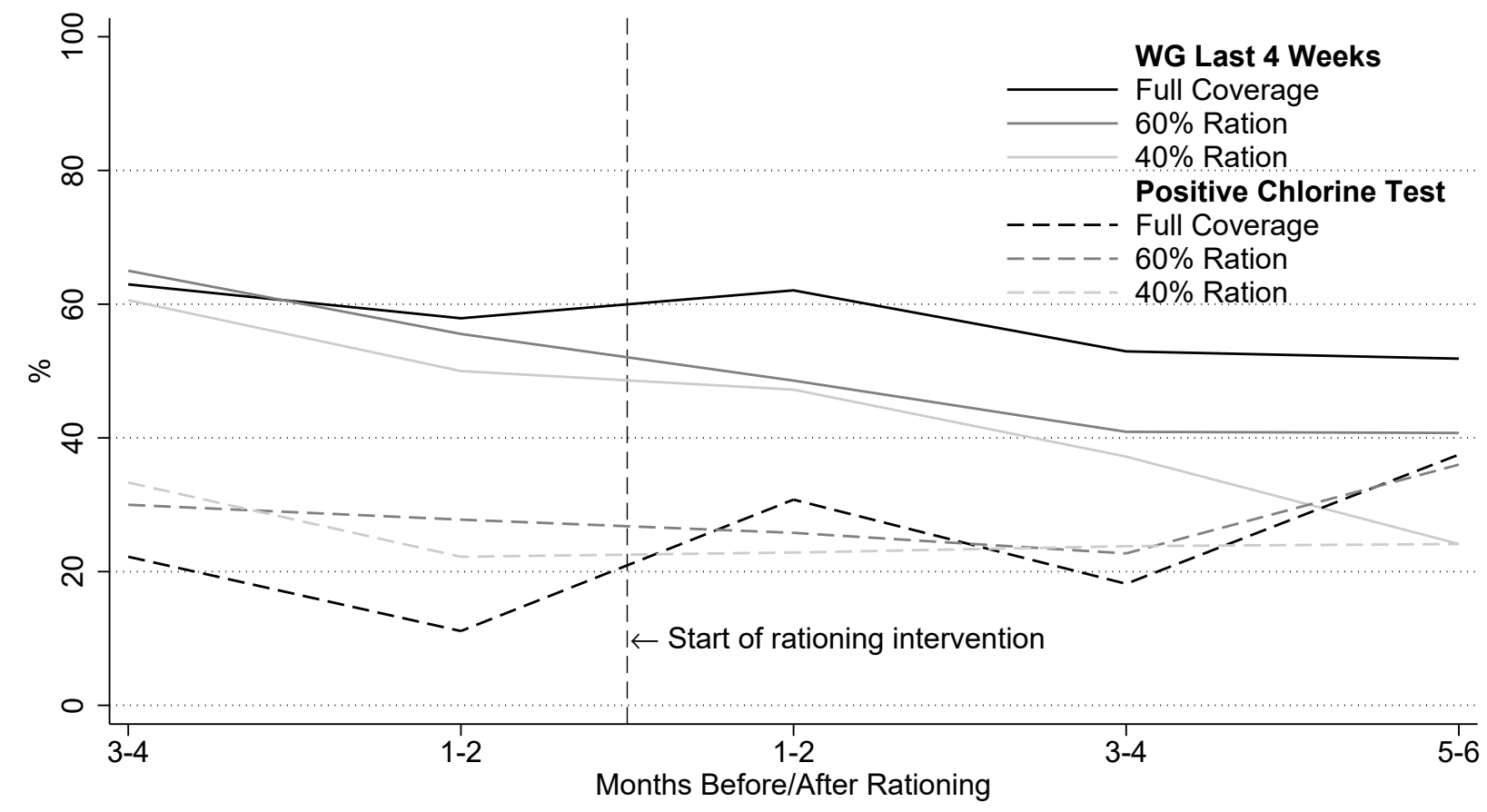

Notes: Dashed lines plot the share of surveyed households that had chlorine treated water (tested by the study team) for the 4 months prior to the start of rationing (February 2019) and 6 months while rationing was ongoing. Shares are averaged across two months to reduce noise. Solid lines plot the share of surveyed households that reported receiving a free bottle of WaterGuard in the previous 4 weeks. 
Figure 5: Heterogeneity in effect sizes, by whether water source is protected

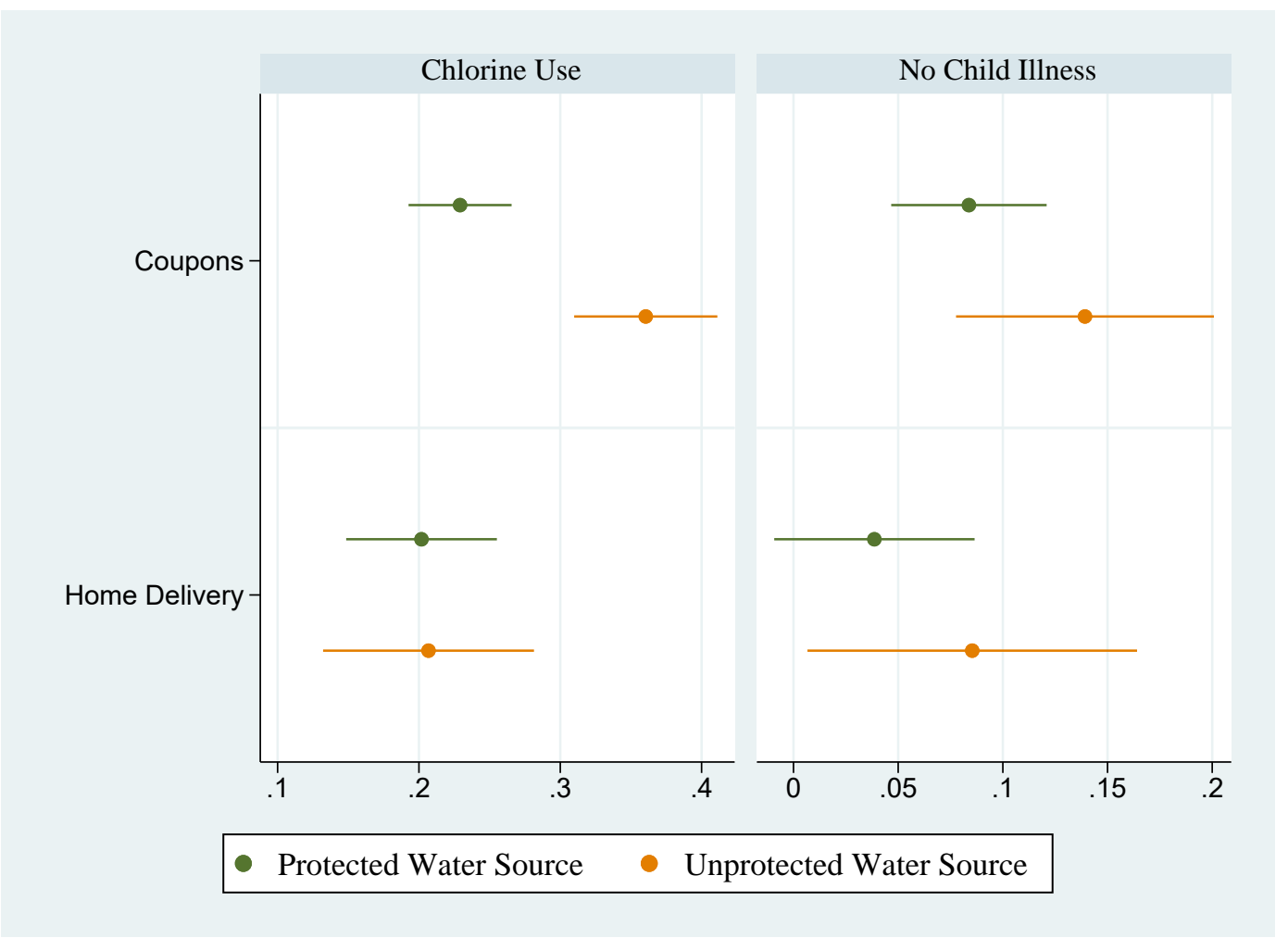

Notes: Estimates are from separate regressions that subset on households based on whether they had a protected water source. Regressions include indicators for Coupon and Home Delivery assignment. Child illness was measured within the previous month for children under 10 years old. The illness effects sizes are the absolute value of the any illness coefficients (positive means less illness). Protected source includes piped water, public tap, borehole with handpump, protected well, and protected spring. Points represent the effect size relative to the control group and error bars are 95\% confidence intervals. Standard errors are clustered at the CHW level in Neno, and at the household level in Mwanza. For Coupons, differences in effect sizes are significant for chlorine use (tested using interaction terms), but not child illness. Differences in effect sizes are not significant for Home Delivery. 
Table 1: Description of sample

\begin{tabular}{lccc}
\hline \hline Variables & Mean & Std. Dev. & $\begin{array}{c}\text { P-Value of Joint } \\
\text { Test for } \\
\text { Orthogonality }\end{array}$ \\
\hline Demographics & & & \\
Age & 30.2 & 9.10 & 0.703 \\
Education (None) & 0.104 & 0.306 & 0.453 \\
Education (Standard 1/4) & 0.240 & 0.427 & 0.993 \\
Education (Standard 5/8) & 0.475 & 0.499 & 0.943 \\
Education (Secondary+) & 0.179 & 0.383 & 0.967 \\
Wealth Index & -.00 & 2.37 & 0.418 \\
Household Size & 5.15 & 1.82 & 0.746 \\
Age of Youngest Child & 2.30 & 1.47 & 0.275 \\
& & & \\
Chlorine Beliefs and Use & & & 0.212 \\
Ever Used WaterGuard & 0.513 & 0.499 & 0.266 \\
Believes Chlorine Makes Water Safe & 0.714 & 0.451 & 0.258 \\
Believes Chlorine Makes Water Taste Bad & 0.462 & 0.498 & 0.095 \\
Positive Chlorine Test & 0.046 & 0.209 & \\
Water Source & & & 0.301 \\
Any Protected Source & & & 0.077 \\
Protected: Piped & 0.706 & 0.455 & 0.405 \\
Protected: Borehole & 0.036 & 0.187 & 0.906 \\
Protected: Well or Spring & 0.647 & 0.477 & 0.445 \\
Believes Own Water Source Always Safe & 0.021 & 0.145 & 0.619 \\
HSA Interaction & 0.504 & 0.500 & \\
HSA gave water management advice & & & 0.763 \\
HSA dispenses WaterGaurd & 0.511 & 0.499 & 0.203 \\
HSA gave free chlorine & 0.043 & 0.270 & \\
\hline \hline
\end{tabular}

Notes: Data source: Baseline surveys conducted between March and April 2018. P-value of joint test for orthogonality was estimated using F-test after regressing the variable on indicators for treatment assignment: coupon, WASH, coupon X WASH, home delivery, home delivery X Rationing, Coupon X Mwanza, and Mwanza. Mwanza was omitted from the F-test. Wealth Index was created using principle components analysis based on household assets. 
Table 2: Impacts on WaterGuard adoption and child health

\begin{tabular}{|c|c|c|c|c|c|}
\hline VARIABLES & $\begin{array}{c}(1) \\
\text { Positive } \\
\text { Chlorine } \\
\text { Test }\end{array}$ & $\begin{array}{c}(2) \\
\text { Self- } \\
\text { Reported } \\
\text { Chlorine } \\
\text { Use }\end{array}$ & $\begin{array}{c}(3) \\
\text { Gave Wa- } \\
\text { terGuard } \\
\text { Away }\end{array}$ & $\begin{array}{l}\text { Any Child } \\
\text { Illness }\end{array}$ & $\begin{array}{c}\text { Number of } \\
\text { Illnesses }\end{array}$ \\
\hline Coupon & $\begin{array}{c}0.259^{* * *} \\
(0.0270)\end{array}$ & $\begin{array}{c}0.304^{* * *} \\
(0.0327)\end{array}$ & $\begin{array}{c}0.124^{* * *} \\
(0.0204)\end{array}$ & $\begin{array}{c}-0.0838 * * * \\
(0.0298)\end{array}$ & $\begin{array}{c}-0.184^{* *} \\
(0.0841)\end{array}$ \\
\hline Coupon X Mwanza & $\begin{array}{c}0.0608 \\
(0.0396)\end{array}$ & $\begin{array}{c}0.0410 \\
(0.0443)\end{array}$ & $\begin{array}{c}0.0326 \\
(0.0309)\end{array}$ & $\begin{array}{l}-0.0120 \\
(0.0449)\end{array}$ & $\begin{array}{l}-0.110 \\
(0.126)\end{array}$ \\
\hline WASH & $\begin{array}{l}0.00746 \\
(0.0145)\end{array}$ & $\begin{array}{c}0.0326^{* *} \\
(0.0144)\end{array}$ & $\begin{array}{l}-0.00797 \\
(0.00838)\end{array}$ & $\begin{array}{c}-0.00744 \\
(0.0287)\end{array}$ & $\begin{array}{c}0.0215 \\
(0.0772)\end{array}$ \\
\hline Coupon X WASH & $\begin{array}{l}-0.0164 \\
(0.0351)\end{array}$ & $\begin{array}{l}-0.0163 \\
(0.0402)\end{array}$ & $\begin{array}{c}0.0537^{* *} \\
(0.0268)\end{array}$ & $\begin{array}{l}-0.0125 \\
(0.0374)\end{array}$ & $\begin{array}{l}-0.135 \\
(0.112)\end{array}$ \\
\hline Home Delivery & $\begin{array}{l}0.195^{* * * *} \\
(0.0259)\end{array}$ & $\begin{array}{l}0.239^{* * *} \\
(0.0262)\end{array}$ & $\begin{array}{c}0.0123 \\
(0.0120)\end{array}$ & $\begin{array}{c}-0.0629^{* *} \\
(0.0299)\end{array}$ & $\begin{array}{l}-0.162^{*} \\
(0.0861)\end{array}$ \\
\hline Home Delivery X Rationing & $\begin{array}{l}0.00118 \\
(0.0416)\end{array}$ & $\begin{array}{c}0.0315 \\
(0.0428)\end{array}$ & $\begin{array}{l}0.00721 \\
(0.0180)\end{array}$ & $\begin{array}{c}0.0505 \\
(0.0325)\end{array}$ & $\begin{array}{c}0.123 \\
(0.104)\end{array}$ \\
\hline Mwanza & $\begin{array}{l}-0.0152 \\
(0.0134)\end{array}$ & $\begin{array}{l}0.00728 \\
(0.0146)\end{array}$ & $\begin{array}{l}-0.00829 \\
(0.00985)\end{array}$ & $\begin{array}{l}0.0615^{*} \\
(0.0319)\end{array}$ & $\begin{array}{c}0.273^{* * *} \\
(0.0822)\end{array}$ \\
\hline Observations & 3,793 & 3,576 & 3,893 & 6,623 & 6,623 \\
\hline Controls & No & No & No & No & No \\
\hline Quarter FE & Yes & Yes & Yes & Yes & Yes \\
\hline Pooled Coupon Effect & $0.266^{* * *}$ & $0.306^{* * *}$ & $0.156^{* * *}$ & $-0.0922^{* * *}$ & $-0.145^{* * *}$ \\
\hline Total Rationed HD Effect & $0.196^{* * *}$ & $0.270 * * *$ & 0.0195 & -0.0124 & -0.0216 \\
\hline P-value of Coupons vs. HD & 0.0385 & 0.00921 & 0.001 & 0.149 & 0.264 \\
\hline Number of clusters & 847 & 841 & 848 & 844 & 844 \\
\hline Neno control group mean & 0.0401 & 0.0331 & 0.0124 & 0.430 & 0.583 \\
\hline
\end{tabular}

Notes: Data are from followup surveys conducted on a rolling basis between May 2018 and July 2019. The order in which households were surveyed was randomized, with stratification at the CHW level. Households were sampled to be surveyed twice, with an average gap of 6.4 months between the two follow-ups. Child illness was measured within the previous month for children under 10 years old. Any child illness (Column 4) indicates whether the child had any of the illnesses we measure (diarrhea, vomiting, and fever), and was constructed from caretaker reports. Column 5 shows a poisson regression of the count of illnesses reported (0 to 3 ). Child health outcomes control for child's age. The pooled coupon effect is the weighted average of the effect in the three arms with coupons: Neno WASH, Neno no WASH, and Mwanza. The Total Rationed HD effect is the effect of HD under rationing compared to the control. Standard errors clustered at the cluster level in parentheses. A cluster is a CHW catchment's area in Neno and a household in Mwanza. HD stand for Home Delivery. ${ }^{* * *} \mathrm{p}<0.01,{ }^{* *} \mathrm{p}<0.05,{ }^{*} \mathrm{p}<0.1$. 
Table 3: Consistency in WaterGuard use

\begin{tabular}{lcc}
\hline \hline & $(1)$ & $(2)$ \\
VARIABLES & $\begin{array}{c}\text { At least one } \\
\text { Positive } \\
\text { Chlorine Test }\end{array}$ & $\begin{array}{c}\text { Always Positive } \\
\text { for Chlorine }\end{array}$ \\
\hline Coupon & $0.402^{* * *}$ & $0.155^{* * *}$ \\
Coupon X Mwanza & $(0.0399)$ & $(0.0290)$ \\
WASH & 0.0533 & 0.0402 \\
Coupon X WASH & $(0.0589)$ & $(0.0422)$ \\
Home Delivery & 0.0273 & 0.0139 \\
Mwanza & $(0.0258)$ & $(0.0125)$ \\
& -0.0579 & -0.0436 \\
& $(0.0552)$ & $(0.0367)$ \\
Observations & $0.348^{* * *}$ & $0.122^{* * *}$ \\
Pooled Coupon Effect & $(0.0401)$ & $(0.0260)$ \\
p-value of Coupons vs. HD & 0.00784 & -0.0116 \\
Number of clusters (CHWs) & $(0.0269)$ & $(0.00820)$ \\
Neno control group mean & 0.417 & 1,499 \\
\hline \hline
\end{tabular}

Notes: Data are collapsed to the household level and include only households that had at least 2 follow-up surveys after their assigned intervention started. Follow-up surveys were conducted on a rolling basis between May 2018 and July 2019. The order in which households were surveyed was randomized, with stratification at the CHW level. Households were sampled to be surveyed twice, with an average gap of 6.4 months between the two follow-ups. 'At least one Positive chlorine Test' indicates that the household's drinking water had a positive chlorine test during at least one of the follow-up visits. 'Always Positive for Chlorine' indicates that the household had a positive chlorine test at all of the follow-up visits. The pooled coupon effect is the weighted average of the effect in the three arms with coupons: Neno WASH, Neno no WASH, and Mwanza. Standard errors clustered at the cluster level in parentheses. A cluster is a $\mathrm{CHW}$ catchment's area in Neno and a household in Mwanza. *** $\mathrm{p}<0.01$, ** $\mathrm{p}<0.05, * \mathrm{p}<0.1$. 
Table 4: Targeting on baseline needs and preferences

\begin{tabular}{|c|c|c|c|c|c|}
\hline VARIABLES & $\begin{array}{c}\text { (1) } \\
\text { CHW visit } \\
\text { last month }\end{array}$ & $\begin{array}{c}(2) \\
\text { Free WG } \\
\text { last month }\end{array}$ & $\begin{array}{c}(3) \\
\text { Share } \\
\text { Coupons } \\
\text { Redeemed }\end{array}$ & $\begin{array}{c}\text { (4) } \\
\text { CHW visit } \\
\text { last month }\end{array}$ & $\begin{array}{c}(5) \\
\text { Free WG } \\
\text { last month }\end{array}$ \\
\hline Unprotected Water Source & $\begin{array}{c}-0.091^{* *} \\
(0.037)\end{array}$ & $\begin{array}{c}0.026 \\
(0.024)\end{array}$ & $\begin{array}{c}19.19^{* * *} \\
(2.49)\end{array}$ & & \\
\hline Home Delivery X Unprotected Source & $\begin{array}{l}-0.042 \\
(0.063)\end{array}$ & $\begin{array}{l}-0.079 \\
(0.078)\end{array}$ & & & \\
\hline Rationing X Unprotected Source & $\begin{array}{l}-0.041 \\
(0.104)\end{array}$ & $\begin{array}{c}0.079 \\
(0.093)\end{array}$ & & & \\
\hline Coupon X Unprotected Source & $\begin{array}{c}0.063 \\
(0.044)\end{array}$ & $\begin{array}{c}0.073 \\
(0.048)\end{array}$ & & & \\
\hline Home Delivery & $\begin{array}{c}0.134^{* * *} \\
(0.031)\end{array}$ & $\begin{array}{c}0.530^{* * *} \\
(0.040)\end{array}$ & & $\begin{array}{c}0.127^{* * *} \\
(0.040)\end{array}$ & $\begin{array}{c}0.573^{* * *} \\
(0.048)\end{array}$ \\
\hline Rationing & $\begin{array}{l}-0.029 \\
(0.048)\end{array}$ & $\begin{array}{c}-0.160^{* * *} \\
(0.055)\end{array}$ & & $\begin{array}{l}-0.106^{*} \\
(0.056)\end{array}$ & $\begin{array}{c}-0.249^{* * *} \\
(0.065)\end{array}$ \\
\hline Coupon & $\begin{array}{c}0.011 \\
(0.024)\end{array}$ & $\begin{array}{c}0.325^{* * *} \\
(0.026)\end{array}$ & & $\begin{array}{c}0.014 \\
(0.026)\end{array}$ & $\begin{array}{c}0.372^{* * *} \\
(0.029)\end{array}$ \\
\hline Thinks WG makes water taste bad & $\begin{array}{c}0.013 \\
(0.018)\end{array}$ & $\begin{array}{c}-0.048^{* *} \\
(0.020)\end{array}$ & $\begin{array}{c}-7.90 * * * \\
(2.26)\end{array}$ & $\begin{array}{c}0.009 \\
(0.018)\end{array}$ & $\begin{array}{c}-0.040^{* *} \\
(0.020)\end{array}$ \\
\hline Mwanza & & & $\begin{array}{c}8.33^{* * *} \\
(2.70)\end{array}$ & & \\
\hline Far from Trading Center (TC) & & & $\begin{array}{c}-12.78^{* * * *} \\
(2.28)\end{array}$ & $\begin{array}{c}-0.065^{* *} \\
(0.033)\end{array}$ & $\begin{array}{c}0.020 \\
(0.022)\end{array}$ \\
\hline Home Delivery X Far from TC & & & & $\begin{array}{c}0.009 \\
(0.054)\end{array}$ & $\begin{array}{l}-0.135^{*} \\
(0.069)\end{array}$ \\
\hline Rationing X Far from TC & & & & $\begin{array}{l}0.151^{*} \\
(0.087)\end{array}$ & $\begin{array}{c}0.233^{* *} \\
(0.095)\end{array}$ \\
\hline Coupon X Far from TC & & & & $\begin{array}{c}0.037 \\
(0.043)\end{array}$ & $\begin{array}{l}-0.060 \\
(0.047)\end{array}$ \\
\hline Constant & $\begin{array}{c}0.575^{* * * *} \\
(0.026)\end{array}$ & $\begin{array}{c}0.059^{* * *} \\
(0.022)\end{array}$ & $\begin{array}{c}55.18^{* * *} \\
(1.95)\end{array}$ & $\begin{array}{c}0.576^{* * *} \\
(0.028)\end{array}$ & $\begin{array}{c}0.056^{* *} \\
(0.023)\end{array}$ \\
\hline Observations & 2,993 & 1,811 & 838 & 2,992 & 1,810 \\
\hline $\begin{array}{l}\text { Control Group Mean } \\
\text { Mean of Dep. Var }\end{array}$ & 0.602 & 0.0545 & 53.36 & 0.602 & 0.0545 \\
\hline
\end{tabular}

Notes: Protected water source includes piped water, public tap, borehole with hand pump, protected well, and protected spring. Columns 1, 2, 4 and 5 restricted to Neno district and include controls for presence of an ART patient, TB patient and age of youngest child as well as trimester fixed effects. Column 3 is restricted to Coupon sample and is at the household level. Free WaterGuard in last 4-Weeks (columns 2 and 5) is self-reported and available for follow-up 2 only. Far from Trading Center means that distance is greater than median $(1.85 \mathrm{~km})$. "Thinks WG makes water taste bad" indicates if the household agreed or strongly agreed at baseline with the statement "WaterGuard makes water taste bad". Standard errors clustered at cluster level in parentheses. A cluster is a CHW catchment's area in Neno and a household in Mwanza. *** $\mathrm{p}<0.01,{ }^{* *} \mathrm{p}<0.05,{ }^{*} \mathrm{p}<0.1$. 
Table 5: Home Delivery: Rationing results

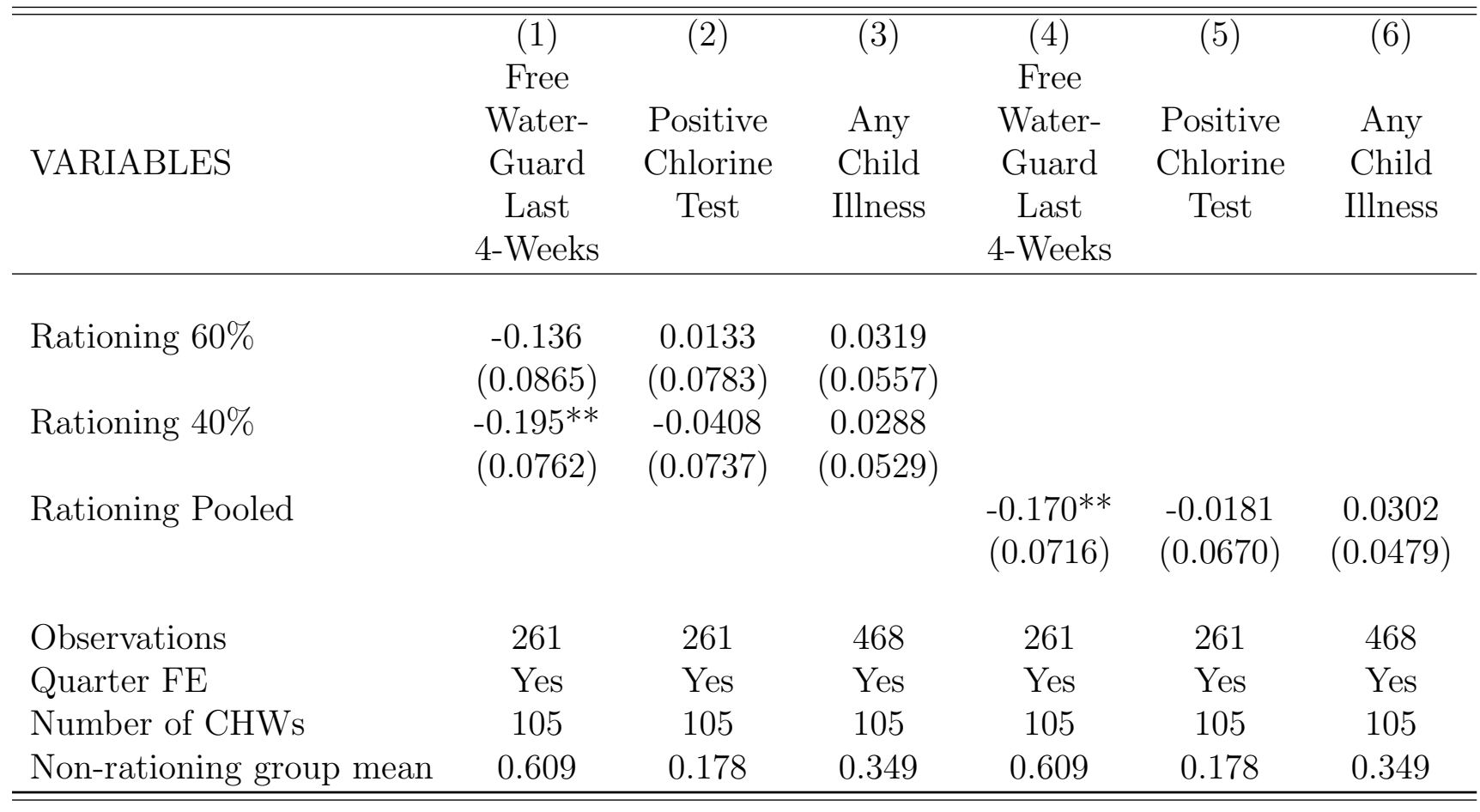

Notes: Data are from follow-up surveys. Sample restricted to Neno households sampled for the Home Delivery treatment and surveyed after the introduction of rationing (February, 12019 ). Columns 4-6 pool 60\% and 40\% rationing levels. Free WaterGuard in last 4-Weeks (columns 1 and 4 ) is self-reported and chlorine tests (columns 2 and 5) were measured using household's water supply. Child illness analysis at the child level (column 6). Child illness was measured within the previous month for children under 10 years old and control for child's age. Standard errors clustered at the cluster level in parentheses. A cluster is a CHW catchment's area in Neno and a household in Mwanza. ${ }^{* * *} \mathrm{p}<0.01,{ }^{* *} \mathrm{p}<0.05,{ }^{*} \mathrm{p}<0.1$. 


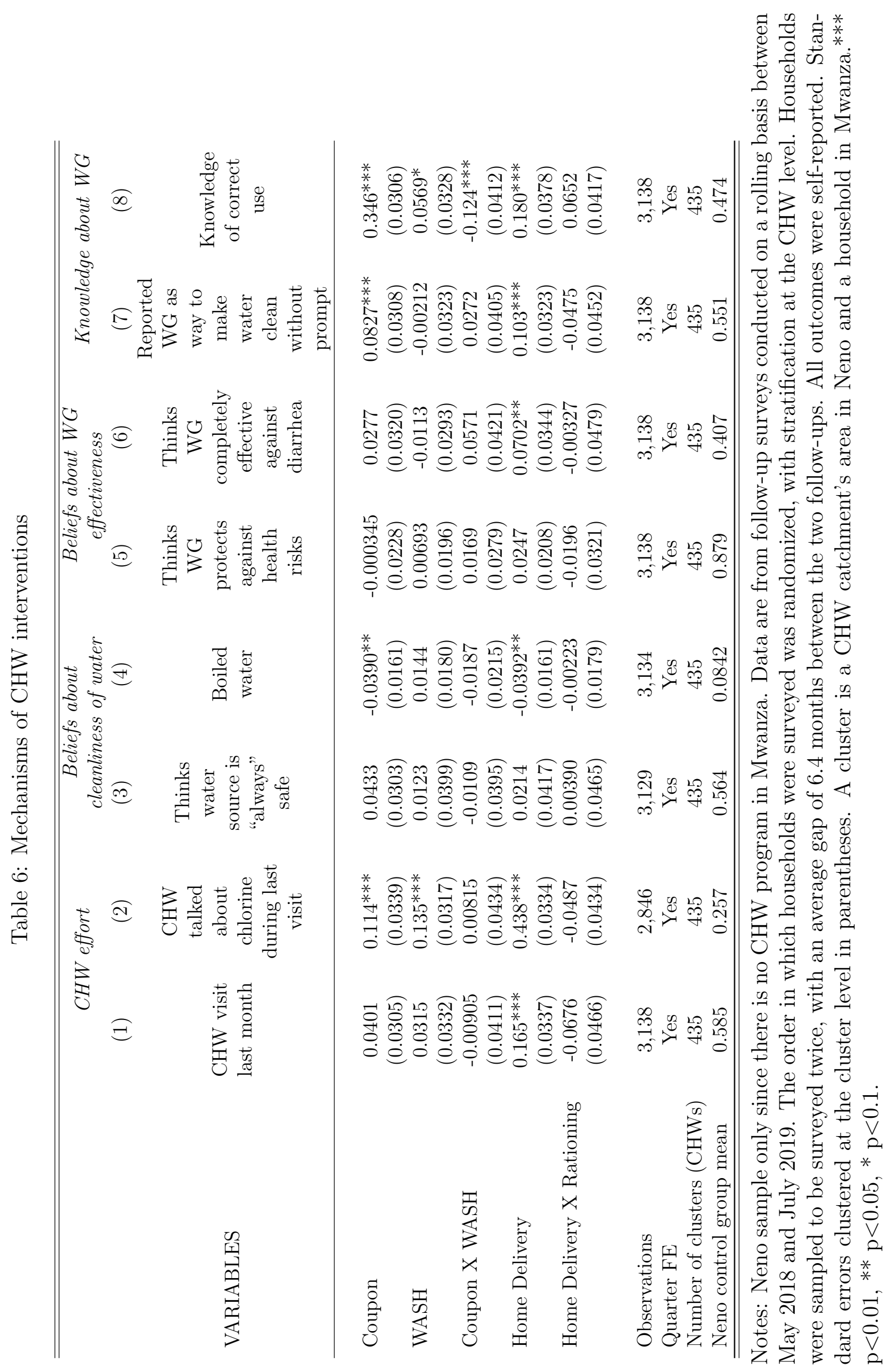


Table 7: Cost-effectiveness

\begin{tabular}{|c|c|c|c|c|c|c|c|}
\hline \multirow{4}{*}{$\begin{array}{l}\text { Coupons } \\
\text { Home Delivery }\end{array}$} & \multicolumn{7}{|c|}{$\begin{array}{l}\text { A. Cost Per Additional Household with Treated Water } \\
\text { (One Month Time Horizon) }\end{array}$} \\
\hline & $\mathrm{N}$ & $\begin{array}{l}\text { Bottles } \\
\text { Dis- } \\
\text { tributed }\end{array}$ & $\begin{array}{l}\text { Cost } \\
\text { Per HH }\end{array}$ & Effect & $\begin{array}{c}\text { Addi- } \\
\text { tional } \\
\text { HH- } \\
\text { days } \\
\text { Treated }\end{array}$ & $\begin{array}{c}\text { Cost per } \\
30 \\
\text { HH-days } \\
\text { Treated }\end{array}$ & $95 \%$ CI \\
\hline & 872 & 419 & $\$ 0.29$ & 0.26 & 6,804 & $\$ 1.13$ & $\$ 1.00-\$ 1.31$ \\
\hline & & & & & & & \\
\hline $40 \%$ Coverage & 1,038 & 415 & $\$ 0.19$ & 0.19 & 5,921 & $\$ 1.05$ & $\$ 0.59-\$ 4.30$ \\
\hline $60 \%$ Coverage & 9,57 & 574 & $\$ 0.29$ & 0.23 & 6,746 & $\$ 1.23$ & $\$ 0.75-\$ 3.40$ \\
\hline \multirow[t]{3}{*}{$100 \%$ Coverage } & 974 & 974 & $\$ 0.47$ & 0.24 & 7,031 & $\$ 1.95$ & $\$ 1.50-\$ 2.60$ \\
\hline & \multicolumn{7}{|c|}{$\begin{array}{l}\text { B. Cost Per Child Illness Averted } \\
\text { (12 Month Time Horizon) }\end{array}$} \\
\hline & $\mathrm{N}$ & $\begin{array}{l}\text { Bottles } \\
\text { Dis- } \\
\text { tributed }\end{array}$ & $\begin{array}{l}\text { Cost } \\
\text { Per HH }\end{array}$ & Effect & $\begin{array}{l}\text { Cases } \\
\text { Averted }\end{array}$ & $\begin{array}{l}\text { Cost per } \\
\text { Case } \\
\text { Averted }\end{array}$ & $95 \% \mathrm{CI}$ \\
\hline Coupons & 872 & 5,027 & $\$ 3.55$ & -0.11 & 1,150 & $\$ 2.69$ & $\$ 2.01-\$ 4.07$ \\
\hline \multicolumn{8}{|l|}{ Home Delivery } \\
\hline $40 \%$ Coverage & 1,038 & 4,983 & $\$ 2.40$ & -0.01 & 104 & $\$ 23.87$ & $\$ 1.77-\mathrm{NA}$ \\
\hline $60 \%$ Coverage & 957 & 6,890 & $\$ 3.48$ & -0.01 & 64 & $\$ 52.17$ & $\$ 2.43-\mathrm{NA}$ \\
\hline $100 \%$ Coverage & 974 & 11,684 & $\$ 5.64$ & -0.04 & 438 & $\$ 12.54$ & $\$ 4.28-\mathrm{NA}$ \\
\hline
\end{tabular}

Notes: We use only the last 12 months of data (September 2018 onward) to reflect steady state costs and effectiveness. Cost-effectiveness (far right columns) is relative to the control group. 95\% CI estimates use the upper and lower end of the $95 \%$ confidence interval of the effect size. NA means that the $95 \%$ confidence interval of the effect size goes through zero. We convert effect sizes to householddays with treated water because we only test a water source on one day. Values in panel A are for one month (averaged across 12 months). Number of households for the coupon arm is all households that received coupons. Number of households in the Home Delivery arms are the total households assigned to Home Delivery including those that were not surveyed. Bottles distributed are the number of coupons redeemed in the coupon arm and number of bottles given to CHWs in Home Delivery arms. Bottles cost $\$ 0.45$ (USD). Distribution of bottles costs $\$ 0.08$ in the coupons arm and $\$ 0.02$ in the delivery arm. Effects for coupon and Home Delivery with $100 \%$ coverage are from Table 2 restricting to the last 12 months. Effects for $60 \%$ and $40 \%$ coverage add the effects for $100 \%$ coverage to the coefficients in Table 5 . 


\section{Appendix A}

Figure A1: Experimental design and sample sizes per treatment arm

\begin{tabular}{|c|c|c|c|}
\hline & \multicolumn{2}{|c|}{ Household Level Randomization } \\
\hline & & Coupons & No Coupons \\
\hline \multirow{2}{*}{ Sample 1: Mwanza } & \multirow{2}{*}{ No CHW } & $\mathrm{N}=220$ & $\mathrm{~N}=220$ \\
\hline & & Households & Households \\
\hline \multirow{3}{*}{$\begin{array}{l}\text { Sample 2: Neno } \\
\text { CHW Randomization }\end{array}$} & $\begin{array}{c}\text { Status quo CHW } \\
(\mathrm{N}=106 \mathrm{CHWs})\end{array}$ & $\begin{array}{c}\mathrm{N}=241 \\
\text { Households }\end{array}$ & $\begin{array}{c}\mathrm{N}=240 \\
\text { Households }\end{array}$ \\
\hline & $\begin{array}{l}\text { WASH Training: CHW } \\
\text { encourages water treatment } \\
\quad(\mathrm{N}=214 \mathrm{CHWs})\end{array}$ & $\begin{array}{c}\mathrm{N}=468 \\
\text { Households }\end{array}$ & $\begin{array}{c}\mathrm{N}=468 \\
\text { Households }\end{array}$ \\
\hline & $\begin{array}{l}\text { Home Delivery: CHW } \\
\text { encourages water treatment } \\
\text { and provides free bottles } \\
(\mathrm{N}=107 \mathrm{CHWs})\end{array}$ & & $\begin{array}{c}\mathrm{N}=458 \\
\text { Households }\end{array}$ \\
\hline
\end{tabular}

Figure A2: Study timeline

\begin{tabular}{|c|c|c|c|c|c|c|c|c|c|c|c|c|c|c|c|c|c|c|}
\hline \multicolumn{19}{|l|}{ Data Collection } \\
\hline \multicolumn{19}{|l|}{ Baseline Survey } \\
\hline \multicolumn{19}{|l|}{ Follow-up Survey Wave 1} \\
\hline \multicolumn{19}{|l|}{ Follow-up Survey Wave 2} \\
\hline \multicolumn{19}{|l|}{ Interventions } \\
\hline \multicolumn{19}{|l|}{ Coupons } \\
\hline \multicolumn{19}{|l|}{ WASH Intervention } \\
\hline \multicolumn{19}{|l|}{ Home Delivery } \\
\hline \multicolumn{19}{|l|}{ Rationing } \\
\hline & Mar & Apr & May & Jun & Jul & Aug & Sep & Oct & Nov & Dec & Jan & Feb & Mar & Apr & May & Jun & Jul & Aug \\
\hline
\end{tabular}


Figure A3: Example wall calendar with attached coupons

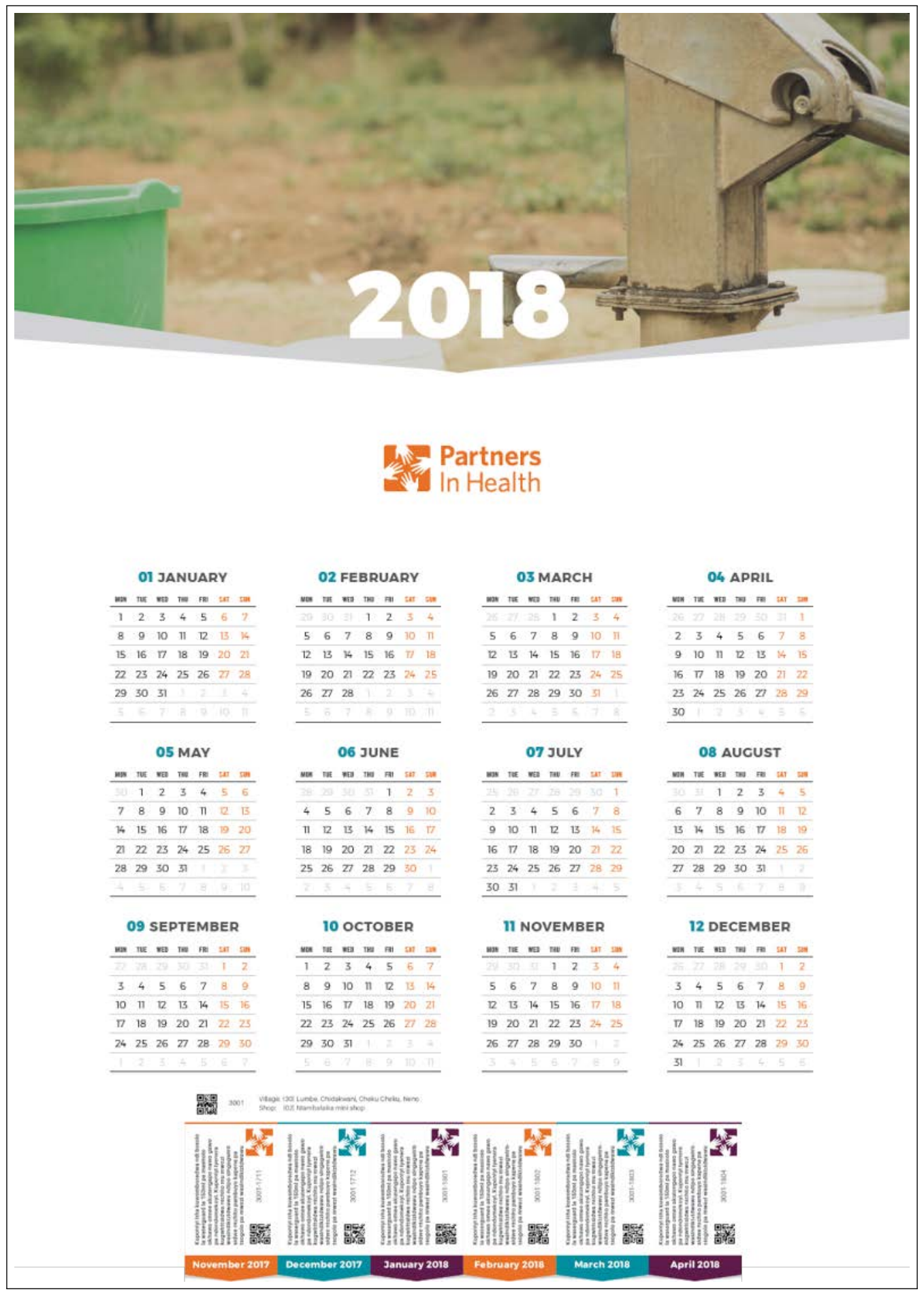

Notes: Households not sampled for coupons received an identical calendar, but without the coupons attached at the bottom. 
Figure A4: Share of children with illness in previous 4 weeks, over time (Home Delivery Compared to Control)

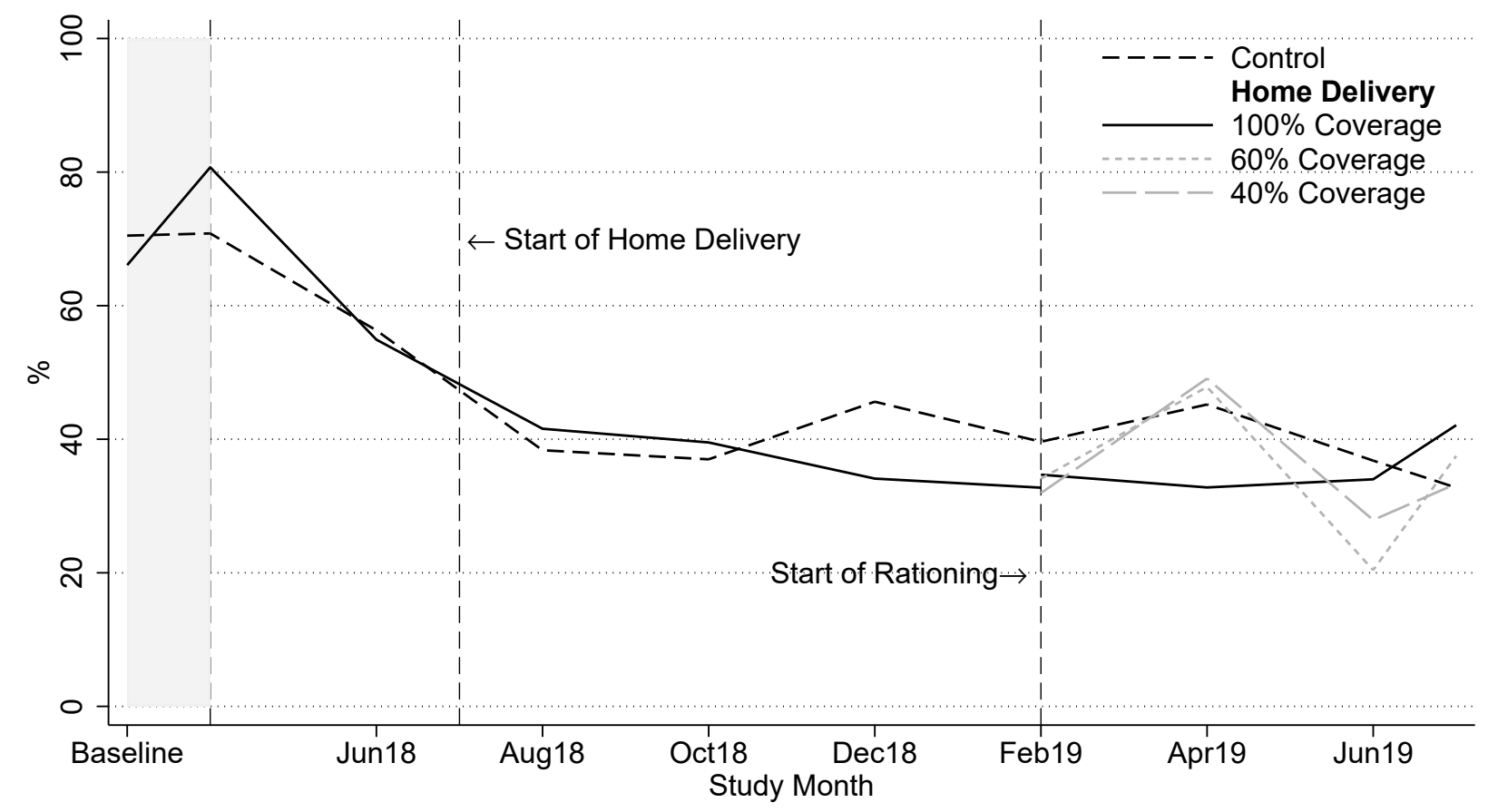

Notes: Lines plot share of children under 10 years old from surveyed households that had at least one of the three illnesses we measure (diarrhea, fever, or vomiting) over 18 months. Time points are averaged across two months to reduce noise (e.g., May and June 2018). An average of 267 households were surveyed each month. 
Figure A5: Distribution of coupon redemption over 18 months

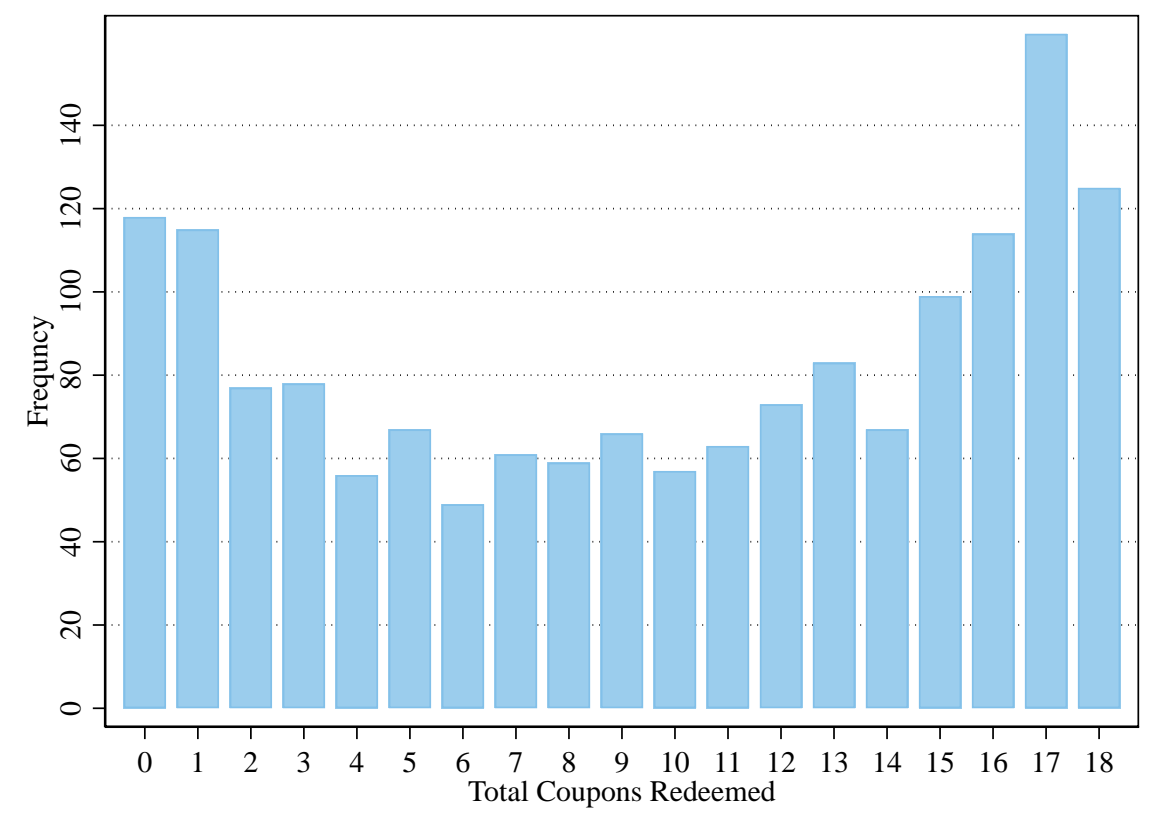

Y-axis is number of households. The $\mathrm{x}$-axis is the number of coupons redeemed. There was a maximum of 18 months of coupon redemption for each household. About 15\% of households did not receive their coupons until the first month had already passed and thus could redeem a maximum of 17. 
Figure A6: Heterogeneity in effect sizes, by whether household thinks WaterGuard makes water taste bad

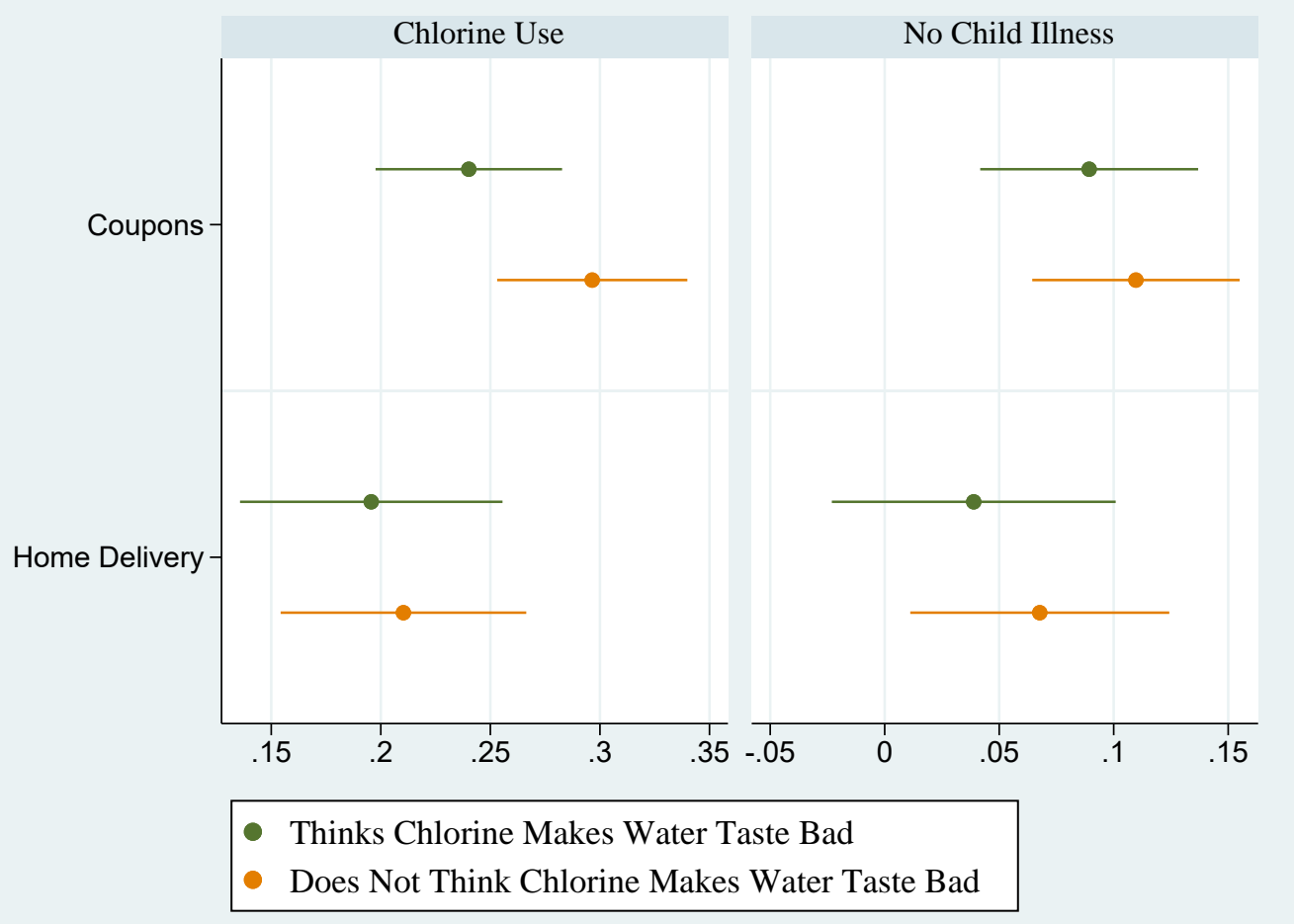

Notes: Estimates are from separate regressions that subset on households based on whether they agreed or strongly agreed that WaterGuard makes water taste bad during the baseline survey. Regressions include indicators for Coupon and Home Delivery assignment. Child illness was measured within the previous month for children under 10 years old. The illness effects sizes are the absolute value of the any illness coefficients (positive means less illness). Points represent the effect size relative to the control group and error bars are 95\% confidence intervals. Standard errors are clustered at the CHW level in Neno, and the household level in Mwanza. For Coupons, the p-value for the difference in effect sizes is 0.07 for chlorine use. Other differences in effect sizes are not significantly different from zero. 
Table A1: CHW characteristics and differences between arms

\begin{tabular}{lccc}
\hline \hline & Mean & $\begin{array}{c}\text { WASH vs. } \\
\text { Control } \\
\text { (std. error) }\end{array}$ & $\begin{array}{c}\text { Free Delivery } \\
\text { vs. Control } \\
\text { (std. error) }\end{array}$ \\
\hline Age & 37.0 & 0.119 & -.28 \\
Female & $(8.70)$ & $(0.998)$ & $(1.08)$ \\
Completed primary school & 0.650 & 0.030 & 0.033 \\
& $(0.477)$ & $(0.056)$ & $(0.064)$ \\
Number of children & 0.653 & -.03 & -.11 \\
& $(0.476)$ & $(0.055)$ & $(0.065)$ \\
Number of households in catchment area & 4.28 & -.43 & -.01 \\
& $(2.13)$ & $(0.265)$ & -.28 \\
Hours per week on CHW activities & 26.8 & -.61 & $(1.42)$ \\
Other job in addition to CHW & $(10.2)$ & $(1.27)$ & 1.29 \\
CHW primary source of income & 10.5 & 0.068 & $(0.745)$ \\
& $(4.72)$ & $(0.500)$ & 0.019 \\
Senior CHW & 0.753 & 0.052 & $(0.062)$ \\
Share of households using chlorine at baseline & $(0.431)$ & $(0.053)$ & -.08 \\
& 0.432 & -.00 & $(0.068)$ \\
\hline \hline
\end{tabular}

Notes: Standard deviation of the mean and standard error of the difference in parentheses. Share of households that used chlorine is the share of households assigned to the CHW whose drinking water tested positive for chlorine residual at baseline. Around 4 households per CHWs were sampled at baseline. 
Table A2: Balance between groups at baseline

\begin{tabular}{lccc}
\hline \hline \multirow{2}{*}{ VARIABLES } & $\begin{array}{c}(1) \\
\text { Positive } \\
\text { Chlorine Test }\end{array}$ & $\begin{array}{c}(2) \\
\text { CHW Visit in } \\
\text { Last 4 Weeks }\end{array}$ & $\begin{array}{c}(3) \\
\text { Any Child } \\
\text { Illness }\end{array}$ \\
\hline & & & \\
Coupon & $-0.0368^{*}$ & -0.0131 & -0.0448 \\
Coupon X Mwanza & $(0.0211)$ & $(0.0373)$ & $(0.0352)$ \\
& 0.0313 & & 0.0107 \\
WASH & $(0.0283)$ & & $(0.0490)$ \\
& -0.0273 & 0.0149 & -0.0153 \\
Coupon X WASH & $(0.0182)$ & $(0.0449)$ & $(0.0318)$ \\
& $0.0659^{* * *}$ & -0.00646 & 0.0124 \\
Home Delivery & $(0.0253)$ & $(0.0483)$ & $(0.0426)$ \\
& -0.0339 & -0.0409 & -0.0195 \\
Home Delivery X Rationing & $(0.0252)$ & $(0.0658)$ & $(0.0495)$ \\
& 0.0399 & -0.00405 & -0.00473 \\
Mwanza & $(0.0249)$ & $(0.0645)$ & $(0.0514)$ \\
& -0.0205 & & $0.115^{* * *}$ \\
Observations & $(0.0212)$ & & $(0.0352)$ \\
Pooled Coupon Effect & & & \\
P-value of joint F-test & 2,083 & 1,756 & 3,664 \\
Number of clusters & 0.00419 & -0.0174 & -0.0361 \\
Neno control group mean & 0.0960 & 0.790 & 0.597 \\
\hline \hline
\end{tabular}

Notes: Data source: Baseline surveys conducted between March and April 2018. Column 3 outcome is at the child level. Standard errors clustered at the cluster level in parentheses. A cluster is a CHW catchment's area in Neno and a household in Mwanza. Child illness was measured within the previous month for children under 10 years old. F-test jointly tests whether the following coefficients are equal to zero: Coupons, WASH Training, Coupons X WASH Training, home delivery, home delivery X Rationing, and Coupons X Mwanza. ${ }^{* * *} \mathrm{p}<0.01,{ }^{* *} \mathrm{p}<0.05,{ }^{*} \mathrm{p}<0.1$. 
Table A3: Health outcomes: breakdown by illness (children under 10)

\begin{tabular}{lcccc}
\hline \hline & $(1)$ & $(2)$ & $(3)$ & $(4)$ \\
VARIABLES & Diarrhea & Fever & Vomit & Cough \\
\hline \multirow{2}{*}{ Coupon } & & & & \\
& -0.0190 & $-0.0687^{* *}$ & -0.0134 & $-0.0734^{* *}$ \\
Coupon X Mwanza & $(0.0169)$ & $(0.0282)$ & $(0.0161)$ & $(0.0308)$ \\
& $-0.0746^{* * *}$ & -0.000205 & -0.0172 & 0.0237 \\
WASH & $(0.0278)$ & $(0.0442)$ & $(0.0281)$ & $(0.0469)$ \\
& 0.00201 & -0.000187 & 0.00689 & 0.00724 \\
Coupon X WASH & $(0.0168)$ & $(0.0275)$ & $(0.0157)$ & $(0.0269)$ \\
& 0.00776 & -0.0358 & -0.0242 & -0.0226 \\
Home Delivery & $(0.0230)$ & $(0.0341)$ & $(0.0206)$ & $(0.0387)$ \\
Home Delivery X Rationing & -0.0206 & $-0.0519 *$ & -0.0156 & $-0.0642^{* *}$ \\
Mwanza & $(0.0170)$ & $(0.0285)$ & $(0.0166)$ & $(0.0324)$ \\
Child Age (Years) & $(0.0243$ & 0.0330 & 0.00465 & 0.0509 \\
& $0.0720^{* * *}$ & $(0.0304)$ & $(0.0176)$ & $(0.0391)$ \\
Pobservations & $(0.0218)$ & $(0.0321)$ & $(0.0210)$ & $(0.0318)$ \\
Number of clusters (CHWs) & $-0.0352^{* * *}$ & $-0.0116^{* * *}$ & $-0.00574^{* * *}$ & $-0.0215^{* * *}$ \\
Neno control group mean & 0.124 & 0.352 & 0.106 & 0.454 \\
\hline \hline
\end{tabular}

Notes: Data are from followup surveys conducted on a rolling basis between May 2018 and July 2019. The order in which households were surveyed was randomized, with stratification at the CHW level. Households were sampled to be surveyed twice, with an average gap of 6.4 months between the two follow-ups. Child illness was measured within the previous month for children under 10 years old. The results restricting the sample to under 5 years old are shown in B4. The pooled coupon effect is the weighted average of the effect in the three arms with coupons: Neno WASH, Neno no WASH, and Mwanza. Standard errors clustered at the cluster level in parentheses. A cluster is a CHW catchment's area in Neno and a household in Mwanza. $\mathrm{p}<0.01,{ }^{* *} \mathrm{p}<0.05,{ }^{*} \mathrm{p}<0.1$. 
Table A4: Association between beliefs about water cleanliness and child illness (Control group)

\begin{tabular}{|c|c|c|c|c|c|c|}
\hline VARIABLES & $\begin{array}{c}(1) \\
\text { Any } \\
\text { Child } \\
\text { Illness }\end{array}$ & $\begin{array}{c}(2) \\
\text { Any } \\
\text { Child } \\
\text { Illness }\end{array}$ & $\begin{array}{c}(3) \\
\text { Any } \\
\text { Child } \\
\text { Illness }\end{array}$ & $\begin{array}{c}(4) \\
\text { Any } \\
\text { Child } \\
\text { Illness }\end{array}$ & $\begin{array}{c}(5) \\
\text { Any } \\
\text { Child } \\
\text { Illness }\end{array}$ & $\begin{array}{c}(6) \\
\text { Any } \\
\text { Child } \\
\text { Illness }\end{array}$ \\
\hline Water always safe & $\begin{array}{c}-0.084^{* * *} \\
(0.015)\end{array}$ & $\begin{array}{c}-0.077^{* * *} \\
(0.015)\end{array}$ & $\begin{array}{c}-0.071^{* * *} \\
(0.016)\end{array}$ & & & \\
\hline Mwanza & & $\begin{array}{c}0.075^{* * *} \\
(0.018)\end{array}$ & $\begin{array}{c}0.076^{* * *} \\
(0.019)\end{array}$ & & $\begin{array}{c}0.085^{* * *} \\
(0.018)\end{array}$ & $\begin{array}{c}0.085^{* * *} \\
(0.019)\end{array}$ \\
\hline Age & & & $\begin{array}{c}0.001 \\
(0.001)\end{array}$ & & & $\begin{array}{c}0.001 \\
(0.001)\end{array}$ \\
\hline Education: Primary 1-4 & & & $\begin{array}{c}0.024 \\
(0.032)\end{array}$ & & & $\begin{array}{c}0.013 \\
(0.032)\end{array}$ \\
\hline Education: Primary 5-8 & & & $\begin{array}{c}0.015 \\
(0.027)\end{array}$ & & & $\begin{array}{c}0.002 \\
(0.027)\end{array}$ \\
\hline Education: Secondary+ & & & $\begin{array}{c}0.009 \\
(0.023)\end{array}$ & & & $\begin{array}{l}-0.001 \\
(0.024)\end{array}$ \\
\hline Wealth Index & & & $\begin{array}{c}-0.011^{* * *} \\
(0.004)\end{array}$ & & & $\begin{array}{c}-0.010^{* * *} \\
(0.004)\end{array}$ \\
\hline Household Size & & & $\begin{array}{l}0.009^{*} \\
(0.005)\end{array}$ & & & $\begin{array}{l}0.008^{*} \\
(0.005)\end{array}$ \\
\hline Child Age & & & $\begin{array}{l}-0.009 \\
(0.006)\end{array}$ & & & $\begin{array}{l}-0.008 \\
(0.006)\end{array}$ \\
\hline Protected Source & & & & $\begin{array}{c}-0.092^{* * *} \\
(0.016)\end{array}$ & $\begin{array}{c}-0.092^{* * *} \\
(0.016)\end{array}$ & $\begin{array}{c}-0.080^{* * * *} \\
(0.017)\end{array}$ \\
\hline Constant & $\begin{array}{c}0.586^{* * *} \\
(0.011)\end{array}$ & $\begin{array}{c}0.566^{* * *} \\
(0.012)\end{array}$ & $\begin{array}{c}0.491^{* * *} \\
(0.038)\end{array}$ & $\begin{array}{c}0.606^{* * *} \\
(0.014)\end{array}$ & $\begin{array}{c}0.587^{* * *} \\
(0.014)\end{array}$ & $\begin{array}{c}0.516^{* * *} \\
(0.039)\end{array}$ \\
\hline Observations & 4,183 & 4,183 & 4,106 & 4,177 & 4,177 & 4,106 \\
\hline
\end{tabular}

Notes: Water Always Safe is from a survey question asking respondents how often their primary water source is safe to drink. Protected source includes piped water, public tap, borehole with handpump, protected well, and protected spring. Child illness was measured for children under 10 years old using caretaker reports of diarrhea, vomiting, and fever in the previous 4 -weeks. Robust standard errors in parentheses. ${ }^{* * *} \mathrm{p}<0.01,{ }^{* *} \mathrm{p}<0.05,{ }^{*} \mathrm{p}<0.1$. 
Table A5: Testing for differential attrition across study arms

\begin{tabular}{lc}
\hline \hline & $(1)$ \\
VARIABLES & Number of Follow-up Visits \\
\hline & 0.027 \\
Coupon & $(0.034)$ \\
Coupon X Mwanza & -0.026 \\
& $(0.047)$ \\
WASH & 0.022 \\
& $(0.030)$ \\
Coupon X WASH & -0.008 \\
& $(0.041)$ \\
Home Delivery & 0.000 \\
& $(0.032)$ \\
Mwanza & 0.054 \\
& $(0.034)$ \\
Observations & 2,307 \\
Controls & No \\
Pooled Coupon Effect & 0.0292 \\
P-value of Coupons vs. HD & 0.135 \\
Number of clusters (CHWs) & 874 \\
Neno control group mean & 1.646 \\
\hline \hline
\end{tabular}

Notes: Estimates are from a poisson regression model that regresses the number of follow-up visits for each household on treatment assignment. Most households had the potential for 2 follow-up visits, but 73 randomly selected households had 3 follow-up visits due to extra resources remaining after 2 follow-up waves were completed. HD stands for Home Delivery. 
Table A6: Balance between groups on baseline characteristics of attriters

\begin{tabular}{lccc}
\hline \hline \multirow{2}{*}{ VARIABLES } & $\begin{array}{c}(1) \\
\text { Positive } \\
\text { Chlorine Test }\end{array}$ & $\begin{array}{c}(2) \\
\text { CHW Visit in } \\
\text { Last 4 Weeks }\end{array}$ & $\begin{array}{c}(3) \\
\text { Any Child } \\
\text { Illness }\end{array}$ \\
\hline & -0.0272 & -0.0450 & -0.0852 \\
Coupon & $(0.0304)$ & $(0.0907)$ & $(0.0670)$ \\
WASH & 0.0101 & -0.0150 & -0.0513 \\
& $(0.0317)$ & $(0.0741)$ & $(0.0570)$ \\
Coupon X WASH & 0.0467 & 0.0388 & 0.0326 \\
Home Delivery & $(0.0409)$ & $(0.110)$ & $(0.0830)$ \\
& $-0.0441^{*}$ & -0.153 & -0.0359 \\
Home Delivery X Rationing & $(0.0245)$ & $(0.0979)$ & $(0.0780)$ \\
& $0.0440^{* *}$ & 0.122 & -0.0347 \\
Mwanza & $(0.0205)$ & $(0.0917)$ & $(0.0793)$ \\
Coupon X Mwanza & 0.0171 & & $0.109^{*}$ \\
& $(0.0423)$ & & $(0.0653)$ \\
Observations & 0.00516 & & -0.0538 \\
Pooled Coupon Effect & $(0.0535)$ & & $(0.0986)$ \\
P-value of joint F-test & & & \\
Number of clusters & -0.00158 & -0.0192 & $-0.0765^{* *}$ \\
Neno control group mean & 0.00217 & 0.715 & 0.301 \\
\hline \hline
\end{tabular}

Notes: Regressions use baseline data from households that have fewer than 2 follow-up visits. Column 3 outcome is at the child level. Standard errors clustered at the cluster level in parentheses. A cluster is a CHW catchment's area in Neno and a household in Mwanza. Child illness was measured within the previous month for children under 10 years old. F-test jointly tests whether the following coefficients are equal to zero: Coupons, WASH Training, Coupons X WASH Training, home delivery, home delivery $\mathrm{X}$ Rationing, and Coupons X Mwanza. 
Table A7: Lower bound Coupon and Home Delivery treatment effects (sensitivity to attrition)

\begin{tabular}{|c|c|c|c|c|}
\hline VARIABLES & $\begin{array}{c}(1) \\
\text { Chlorine: } \\
\text { No } \\
\text { Attrition }\end{array}$ & $\begin{array}{c}(2) \\
\text { Chlorine: } \\
\text { With } \\
\text { Attrition }\end{array}$ & $\begin{array}{l}\text { (3) } \\
\text { Any Child } \\
\text { Illness: No } \\
\text { Attrition }\end{array}$ & $\begin{array}{c}(4) \\
\text { Any Child } \\
\text { Illness: } \\
\text { With } \\
\text { Attrition }\end{array}$ \\
\hline Coupon & $\begin{array}{c}0.250^{* * *} \\
(0.0179)\end{array}$ & $\begin{array}{c}0.0392^{* *} \\
(0.0198)\end{array}$ & $\begin{array}{c}-0.0989 * * * \\
(0.0185)\end{array}$ & $\begin{array}{c}0.0130 \\
(0.0181)\end{array}$ \\
\hline Coupon X Mwanza & $\begin{array}{c}0.0685^{* *} \\
(0.0340)\end{array}$ & $\begin{array}{c}0.0899^{* *} \\
(0.0371)\end{array}$ & $\begin{array}{c}0.00496 \\
(0.0388)\end{array}$ & $\begin{array}{l}0.00173 \\
(0.0373)\end{array}$ \\
\hline Home Delivery & $\begin{array}{c}0.186^{* * *} \\
(0.0252)\end{array}$ & $\begin{array}{c}-0.000890 \\
(0.0246)\end{array}$ & $\begin{array}{c}-0.0698 * * * \\
(0.0234)\end{array}$ & $\begin{array}{c}0.0186 \\
(0.0231)\end{array}$ \\
\hline Mwanza & $\begin{array}{l}-0.0153 \\
(0.0120)\end{array}$ & $\begin{array}{l}-0.0387^{*} \\
(0.0233)\end{array}$ & $\begin{array}{c}0.0552^{* *} \\
(0.0270)\end{array}$ & $\begin{array}{l}0.0523^{* *} \\
(0.0254)\end{array}$ \\
\hline childage_years & & & $\begin{array}{c}-0.0332^{* * *} \\
(0.00277)\end{array}$ & $\begin{array}{c}-0.0313^{* * *} \\
(0.00279)\end{array}$ \\
\hline Observations & 3,614 & 4,289 & 6,305 & 6,981 \\
\hline Pooled Coupon Effect & $0.267 * * *$ & $0.0602^{* * *}$ & $-0.0979 * * *$ & 0.0134 \\
\hline P-value of Coupons vs. HD & 0.0259 & 0.115 & 0.222 & 0.811 \\
\hline Number of clusters (CHWs) & 847 & 874 & 844 & 873 \\
\hline Neno control group mean & 0.0401 & 0.218 & 0.430 & 0.381 \\
\hline
\end{tabular}

Notes: Estimates are based on the same regression equations as table 2 but without the rationing arm and without quarter fixed effects. The sample without attrition includes all households for which outcome data was collected. The sample with attrition adds synthetic observations for all households with a baseline survey that did not have follow-up data in one or both follow-up waves. Observations in the control arm with missing surveys were all assumed to have had good outcomes (treated water and no illness) and observations from the treatment arms (Coupons and Home Delivery) were all assumed to have had bad outcomes (no treated water and illness. We exclude the WASH treatment from this exercise for simplicity since it had not effect. 
Table A8: Cost-effectiveness (diarrhea cases averted)

\begin{tabular}{lccccccc}
\hline \hline & $\mathrm{N}$ & $\begin{array}{c}\text { Bottles } \\
\text { Dis- } \\
\text { tributed }\end{array}$ & $\begin{array}{c}\text { Cost } \\
\text { Per HH }\end{array}$ & Effect & $\begin{array}{c}\text { Cases } \\
\text { Averted }\end{array}$ & $\begin{array}{c}\text { Cost per } \\
\text { Illness } \\
\text { Averted }\end{array}$ & $95 \%$ CI \\
Coupons & 872 & 5,027 & $\$ 3.55$ & -0.05 & 484 & $\$ 6.40$ & $\$ 4.27-\$ 12.80$ \\
$\begin{array}{l}\text { Home Delivery } \\
40 \% \text { Coverage }\end{array}$ & 1,038 & 4,983 & $\$ 2.40$ & 0.00 & 58 & $\$ 43.02$ & $\$ 3.00-\mathrm{NA}$ \\
$60 \%$ Coverage & 957 & 6,890 & $\$ 3.48$ & 0.02 & -206 & $\mathrm{NA}$ & $\$ 4.89-\mathrm{NA}$ \\
$100 \%$ Coverage & 974 & 11,684 & $\$ 5.64$ & -0.02 & 227 & $\$ 24.22$ & $\$ 7.89-\mathrm{NA}$ \\
\hline \hline
\end{tabular}

Notes: We use only the last 12 months of data (September 2018 onward) to reflect steady state costs and effectiveness. Cost-effectiveness (far right columns) is relative to the control group. 95\% CI estimates use the upper and lower end of the $95 \%$ confidence interval of the effect size. NA means that the $95 \%$ confidence interval of the effect size goes through zero or the effect on diarrhea cases is positive. Number of households for the coupon arm is all households that received coupons. Number of households in the Home Delivery arms are the total households assigned to Home Delivery including those that were not surveyed. Bottles distributed are the number of coupons redeemed in the coupon arm and number of bottles given to CHWs in Home Delivery arms. Bottles cost $\$ 0.45$ (USD). Distribution of bottles costs $\$ 0.08$ in the coupons arm and $\$ 0.02$ in the delivery arm. Effect sizes for coupon and Home Delivery with 100\% coverage are from Table A3 regressions restricting to the last 12 months. Effects for $60 \%$ and $40 \%$ coverage add the effects for $100 \%$ coverage to the rationing coefficients. 


\section{Appendix B: Robustness Checks}

Table B1: Robustness to coding water treatment variable (coding no water as missing)

\begin{tabular}{lc}
\hline \hline VARIABLES & $\begin{array}{c}(1) \\
\text { Positive } \\
\text { Chlorine } \\
\text { Test }\end{array}$ \\
\hline & $0.267^{* * *}$ \\
Coupon & $(0.0277)$ \\
& $0.0739^{*}$ \\
Coupon X Mwanza & $(0.0410)$ \\
& 0.00764 \\
WASH & $(0.0149)$ \\
& -0.0107 \\
Coupon X WASH & $(0.0362)$ \\
& $0.202^{* * *}$ \\
Home Delivery & $(0.0263)$ \\
& 0.00445 \\
Home Delivery X Rationing & $(0.0433)$ \\
& -0.0164 \\
Mwanza & $(0.0138)$ \\
& 3,635 \\
Observations & Yes \\
Quarter FE & $0.280^{* * *}$ \\
Pooled Coupon Effect & 0.0289 \\
P-value of Coupons vs. HD & 837 \\
Number of clusters (CHWs) & 0.0417 \\
Neno control group mean & \\
\hline \hline Anaylsis is identical to column 1 of table 2, \\
but households with no drinking water were \\
excluded rather than coded as not having \\
treated water. This excludes about $5 \%$ of \\
observations.
\end{tabular}


Table B2: Impacts on WaterGuard adoption and child health (controlling for outcomes at baseline)

\begin{tabular}{|c|c|c|c|c|c|}
\hline VARIABLES & $\begin{array}{c}(1) \\
\text { Positive } \\
\text { Chlorine } \\
\text { Test }\end{array}$ & $\begin{array}{c}(2) \\
\text { Self- } \\
\text { Reported } \\
\text { Chlorine } \\
\text { Use } \\
\end{array}$ & $\begin{array}{c}(3) \\
\text { Gave } \\
\text { Water- } \\
\text { Guard } \\
\text { Away } \\
\end{array}$ & $\begin{array}{c}\text { Any Child } \\
\text { Illness }\end{array}$ & $\begin{array}{c}\text { Number of } \\
\text { Illnesses }\end{array}$ \\
\hline Coupon & $\begin{array}{c}0.263^{* * *} \\
(0.0269)\end{array}$ & $\begin{array}{c}0.309 * * * \\
(0.0332)\end{array}$ & $\begin{array}{c}0.126^{* * *} \\
(0.0214)\end{array}$ & $\begin{array}{c}-0.0807^{* * *} \\
(0.0307)\end{array}$ & $\begin{array}{c}-0.180 * * \\
(0.0851)\end{array}$ \\
\hline Coupon X Mwanza & $\begin{array}{c}0.0661^{*} \\
(0.0397)\end{array}$ & $\begin{array}{c}0.0494 \\
(0.0449)\end{array}$ & $\begin{array}{c}0.0310 \\
(0.0318)\end{array}$ & $\begin{array}{c}-0.00477 \\
(0.0454)\end{array}$ & $\begin{array}{l}-0.111 \\
(0.125)\end{array}$ \\
\hline WASH & $\begin{array}{l}0.00466 \\
(0.0147)\end{array}$ & $\begin{array}{c}0.0320 * * \\
(0.0148)\end{array}$ & $\begin{array}{l}-0.00878 \\
(0.00886)\end{array}$ & $\begin{array}{l}0.00276 \\
(0.0281)\end{array}$ & $\begin{array}{c}0.0435 \\
(0.0736)\end{array}$ \\
\hline Coupon X WASH & $\begin{array}{l}-0.0189 \\
(0.0354)\end{array}$ & $\begin{array}{l}-0.0199 \\
(0.0409)\end{array}$ & $\begin{array}{c}0.0562 * * \\
(0.0278)\end{array}$ & $\begin{array}{l}-0.0176 \\
(0.0380)\end{array}$ & $\begin{array}{l}-0.107 \\
(0.113)\end{array}$ \\
\hline Home Delivery & $\begin{array}{c}0.192^{* * *} \\
(0.0268)\end{array}$ & $\begin{array}{c}0.238^{* * *} \\
(0.0272)\end{array}$ & $\begin{array}{c}0.0102 \\
(0.0124)\end{array}$ & $\begin{array}{l}-0.0486^{*} \\
(0.0288)\end{array}$ & $\begin{array}{c}-0.113 \\
(0.0802)\end{array}$ \\
\hline Home Delivery X Rationing & $\begin{array}{r}-0.00479 \\
(0.0412)\end{array}$ & $\begin{array}{c}0.0289 \\
(0.0428)\end{array}$ & $\begin{array}{c}0.0109 \\
(0.0186)\end{array}$ & $\begin{array}{c}0.0413 \\
(0.0330)\end{array}$ & $\begin{array}{l}0.0931 \\
(0.102)\end{array}$ \\
\hline Mwanza & $\begin{array}{l}-0.0180 \\
(0.0137)\end{array}$ & $\begin{array}{l}0.00186 \\
(0.0148)\end{array}$ & $\begin{array}{r}-0.00936 \\
(0.0102)\end{array}$ & $\begin{array}{c}0.0451 \\
(0.0320)\end{array}$ & $\begin{array}{c}0.207^{* * *} * \\
(0.0801)\end{array}$ \\
\hline Treated Water (Residual Chlorine) & $\begin{array}{l}0.0686^{*} \\
(0.0371)\end{array}$ & $\begin{array}{c}0.0374 \\
(0.0379)\end{array}$ & $\begin{array}{l}-0.0278 \\
(0.0213)\end{array}$ & $\begin{array}{l}-0.0324 \\
(0.0293)\end{array}$ & $\begin{array}{l}-0.0518 \\
(0.0939)\end{array}$ \\
\hline Any Illness (Baseline) & & & & $\begin{array}{c}0.137^{* * *} \\
(0.0155)\end{array}$ & \\
\hline Number of Illnesses (Baseline) & & & & & $\begin{array}{c}0.270 * * * \\
(0.0230)\end{array}$ \\
\hline Observations & 3,649 & 3,442 & 3,746 & 6,370 & 6,370 \\
\hline Controls & Yes & Yes & Yes & Yes & No \\
\hline Quarter FE & Yes & Yes & Yes & Yes & Yes \\
\hline Pooled Coupon Effect & $0.270^{* * *}$ & $0.312^{* * *}$ & $0.159^{* * *}$ & $-0.0900 * * *$ & $-0.137^{* * *}$ \\
\hline P-value of Coupons vs. HD & 0.0349 & 0.00848 & 0.001 & 0.0649 & 0.158 \\
\hline Number of clusters & 837 & 831 & 838 & 834 & 834 \\
\hline Neno Control Group mean & 0.0401 & 0.0331 & 0.0124 & 0.430 & 0.583 \\
\hline
\end{tabular}

Notes: Baseline controls indicate that we control for the dependent variable at baseline. Data are from followup surveys conducted on a rolling basis between May 2018 and July 2019. The order in which households were surveyed was randomized, with stratification at the CHW level. Households were sampled to be surveyed twice, with an average gap of 6.4 months between the two follow-ups. Child illness was measured within the previous month for children under 10 years old. Any child illness (Column 4) indicates whether the child had any of the illnesses we measure (diarrhea, vomiting, and fever), and was constructed from caretaker reports. Column 5 is a poisson regression of the number of illnesses reported. All child health regressions control for child's age. The pooled coupon effect is the weighted average of the effect in the three arms with coupons: Neno WASH, Neno no WASH, and Mwanza. Standard errors clustered at the cluster level in parentheses. A cluster is a CHW catchment's area in Neno and a househ@ld in Mwanza. ${ }^{* * *} \mathrm{p}<0.01,{ }^{* *} \mathrm{p}<0.05,{ }^{*} \mathrm{p}<0.1$. 
Table B3: Robustness of diarrhea results to different recall duration

\begin{tabular}{lccc}
\hline \hline & $(1)$ & $(2)$ & $(3)$ \\
VARIABLES & $\begin{array}{c}\text { Diarrhea } \\
\text { Case in Last } \\
4 \text { Weeks }\end{array}$ & $\begin{array}{c}\text { Diarrhea } \\
\text { Case in Last } \\
14 \text { days }\end{array}$ & $\begin{array}{c}\text { Diarrhea } \\
\text { Case in Last } \\
7 \text { days }\end{array}$ \\
\hline & & & \\
Coupon & -0.0190 & $-0.0258^{*}$ & -0.0189 \\
& $(0.0169)$ & $(0.0151)$ & $(0.0134)$ \\
Coupon X Mwanza & $-0.0746^{* * *}$ & $-0.0522^{* *}$ & $-0.0462^{* *}$ \\
& $(0.0278)$ & $(0.0246)$ & $(0.0218)$ \\
WASH & 0.00201 & -0.0127 & -0.00243 \\
& $(0.0168)$ & $(0.0143)$ & $(0.0125)$ \\
Coupon X WASH & 0.00776 & 0.00999 & -0.000344 \\
& $(0.0230)$ & $(0.0191)$ & $(0.0175)$ \\
Home Delivery & -0.0206 & $-0.0286^{*}$ & -0.0199 \\
& $(0.0170)$ & $(0.0154)$ & $(0.0137)$ \\
Home Delivery X Rationing & 0.0243 & 0.0252 & 0.00230 \\
& $(0.0228)$ & $(0.0205)$ & $(0.0166)$ \\
Mwanza & $0.0720^{* * *}$ & $0.0463^{* *}$ & $0.0378^{* *}$ \\
Child Age (Years) & $(0.0218)$ & $(0.0195)$ & $(0.0171)$ \\
& $-0.0352^{* * *}$ & $-0.0264^{* * *}$ & $-0.0205^{* * *}$ \\
Observations & $(0.00206)$ & $(0.00185)$ & $(0.00167)$ \\
Quarter FE & & & \\
Pooled Coupon Effect & 6,628 & 6,628 & 6,628 \\
P-value of Coupons vs. HD & Yes & Yes & Yes \\
Number of clusters (CHWs) & $0.0318^{* * *}$ & $-0.0326^{* * *}$ & $-0.0292^{* * *}$ \\
Neno Control Group Mean & 845 & 0.903 & 0.947 \\
\hline \hline & 0.124 & 845 & 845 \\
& & 0.106 & 0.0806 \\
\hline
\end{tabular}

Notes: Child diarrhea was measured within the previous month for children under 10 years old (Column 1). When a case was reported, a follow-up question asked if the case was ongoing and if not, then how many days ago the case ended. We use these questions to identify diarrhea cases in the previous 14 days and previous 7 days. The pooled coupon effect is the weighted average of the effect in the three arms with coupons: Neno WASH, Neno no WASH, and Mwanza. Standard errors clustered at the cluster level in parentheses. A cluster is a CHW catchment's area in Neno and a household in Mwanza. $\mathrm{p}<0.01,{ }^{* *} \mathrm{p}<0.05,{ }^{*} \mathrm{p}<0.1$ 
Table B4: Health outcomes: breakdown by illness (children under 5)

\begin{tabular}{lcccc}
\hline \hline & $(1)$ & $(2)$ & $(3)$ & $(4)$ \\
VARIABLES & Diarrhea & Fever & Vomit & Cough \\
\hline \multirow{2}{*}{ Coupon } & & & & \\
Coupon X Mwanza & -0.0291 & $-0.0635^{* *}$ & 0.00536 & -0.0563 \\
& $-0.104^{* * *}$ & -0.00671 & $-0.0581^{*}$ & 0.0173 \\
WASH & $(0.0370)$ & $(0.0493)$ & $(0.0337)$ & $(0.0520)$ \\
& 0.00353 & 0.00396 & 0.0188 & 0.0242 \\
Coupon X WASH & $(0.0220)$ & $(0.0298)$ & $(0.0183)$ & $(0.0301)$ \\
& 0.0158 & -0.0468 & -0.0388 & -0.0392 \\
Home Delivery & $(0.0308)$ & $(0.0368)$ & $(0.0239)$ & $(0.0436)$ \\
Home Delivery X Rationing & -0.0225 & -0.0451 & 0.00880 & -0.0615 \\
& $(0.0225)$ & $(0.0326)$ & $(0.0204)$ & $(0.0374)$ \\
Mwanza & 0.0265 & 0.0253 & 0.00136 & 0.0720 \\
Child Age (Years) & $(0.0324)$ & $(0.0403)$ & $(0.0223)$ & $(0.0475)$ \\
& $0.102^{* * *}$ & 0.0472 & $0.100^{* * *}$ & $0.0734^{* *}$ \\
& $(0.0291)$ & $(0.0360)$ & $(0.0255)$ & $(0.0355)$ \\
Observations & $-0.0485^{* * *}$ & 0.00493 & -0.00402 & $-0.0151^{* * *}$ \\
Quarter FE & $(0.00407)$ & $(0.00501)$ & $(0.00350)$ & $(0.00550)$ \\
Pooled Coupon Effect & & & & \\
P-value of Coupons vs. HD & 0.813 & 0.0966 & 0.459 & 0.952 \\
Number of clusters (CHWs) & 819 & 818 & 819 & 819 \\
Neno control group mean & 0.165 & 0.380 & 0.107 & 0.476 \\
\hline \hline
\end{tabular}

Notes: Data are from followup surveys conducted on a rolling basis between May 2018 and July 2019. The order in which households were surveyed was randomized, with stratification at the CHW level. Households were sampled to be surveyed twice, with an average gap of 6.4 months between the two follow-ups. Child illness was measured within the previous month for children from caretaker reports. The pooled coupon effect is the weighted average of the effect in the three arms with coupons: Neno WASH, Neno no WASH, and Mwanza. Standard errors clustered at the cluster level in parentheses. A cluster is a CHW catchment's area in Neno and a household in Mwanza. $\mathrm{p}<0.01,{ }^{* *} \mathrm{p}<0.05,{ }^{*} \mathrm{p}<0.1$. 